\title{
The stability of elastically strained nanorings and the formation of quantum dot molecules
}

\author{
Simon P.A. Gill \\ Department of Engineering, \\ University of Leicester, \\ University Road, \\ Leicester, UK \\ LE1 7RH \\ spg3@le.ac.uk
}

\begin{abstract}
Self-assembled nanorings have recently been identified in a number of heteroepitaxially strained material systems. Under some circumstances these rings have been observed to break up into ringshaped quantum dot molecules. A general non-linear model for the elastic strain energy of nonaxisymmetric epitaxially strained nanostructures beyond the small slope assumption is developed. This model is then used to investigate the stability of strained nanorings evolving via surface diffusion subject to perturbations around their circumference. An expression for the fastest growing mode is determined and related to experimental observations. The model predicts a region of stability for rings below a critical radius, and also a region for larger rings which have a proportionally small thickness. The predictions of the model are shown to be consistent with the available results. For the heteroepitaxial $\operatorname{InP}$ on $\ln _{0.5} \mathrm{Ga}_{0.5} \mathrm{P}$ system investigated by Jevasuwan et al. (2013), the nanorings are found to be stable below a certain critical size. This is in good quantitative agreement with the model predictions. At larger sizes, the rings are unstable. The number of dots in the resulting quantum dot molecule is similar to the mode number for the fastest growing mode. Second order terms show that the number of dots is expected to reduce as the height of the ring increases in proportion to its thickness. The strained $\ln _{0.4} \mathrm{Ga}_{0.6}$ As on $\mathrm{GaAs}$ nanorings of Hanke et al (2007) are always stable and this is in accordance with the findings of the analysis. The Au nanorings of Ruffino et al. (2011) are stable as well, even as they expand during annealing. This observation is also shown to be consistent with the proposed model, which is expected to be useful in the design and tailoring of heteroepitaxial systems for the self-organisation of quantum dot molecules.
\end{abstract}

Keywords: variational calculus; semiconductor material; diffusion, surface; elastic material; nanorings

\section{Introduction}

The unique nature of the electronic confinement in nanorings generates a range of quantum effects with possible exploitation in optical and magnetic applications and quantum computation devices (Ruffino et al, 2011; Somaschini et al, 2009).Their structure-dependent transmission/absorption spectra offer tunable plasmon resonances for optoelectronic devices (Sun et al, 2011; Fang et al, 2007; Kelf et al, 2011) and they have the potential for use in sensing devices (Huang et al, 2012; Aizpurua, 2003) and magnetic data storage devices (Yu et al, 2007). Nanorings have been produced 
by a variety of methods, such as the coating of nanoparticles (Larsson et al, 2007; He at al 2010), the use of templates (Hobbs et al, 2004), the agglomeration of nanoclusters (Mishra et al, 2007), deposition on block co-polymers (Zahr and Blum, 2012; Wang et al, 2011), partial capping of quantum dots (Stoffel et al, 2009) and droplet epitaxy (Somaschini et al, 2009; Li and Yang, 2008). Most of these techniques are strain-free, except for the partial capping process, which typically involves significant intermixing producing a non-uniformly alloyed quantum ring (Hanke et al, 2007). Jevasuwan et al. (2011) have recently developed the technique of strained droplet epitaxy to produce nanorings of pure crystalline $\mathrm{InP}$. In this process, liquid indium droplets are deposited onto an $\mathrm{In}_{0.5} \mathrm{Ga}_{0.5} \mathrm{P}$ substrate, and subsequently exposed to a phosphorus beam. The In and $\mathrm{P}$ combine at the edge of the droplet to form crystalline InP. The InP continues to grow around the perimeter at the expense of the droplet, until the In droplet is entirely consumed leaving a InP nanoring. Above a certain size, the epitaxially strained nanoring becomes unstable (Jevasuwan et al, 2013) leading to the formation of a ring-shaped quantum dot molecule (QDM). This typically generates 6 to 14 quantum dots around the perimeter of the original droplet, depending on the geometry of the original nanoring. This does not occur in unstrained droplet epitaxy, for which the growth mechanisms are well understood (Li and Yang, 2008; Li and Yang, 2009; Zhou et al, 2013). It is therefore reasonable to assume that the large epitaxial mismatch strain present in the InP on $\mathrm{In}_{0.5} \mathrm{Ga}_{0.5} \mathrm{P}$ system is destabilising the nanoring. QDMs also have a variety of potential uses in optoelectronics (Wang et al, 2006) and quantum computation (Amlani et al, 1999; Li et al, 2003). Construction of these devices requires control over the size, shape, number and arrangement of the quantum dots within the QDM. A range of QDMs have been produced by other routes, mostly mediated by strain relief, due to preferential formation of quantum dots around mounds (Lee et al, 2008) or pits (Hu et al, 2012), producing bi-QDMs, quad-QDMs, hexa-QDMs and multi-QDMs.

This paper considers the stability of heteroepitaxial nanorings with the intention of determining the relationship between the geometry of a nanoring and its stability, with the expectation that the dominant unstable mode will determine the number of quantum dots in the resulting QDM. The main complexity of this analysis is determination of the elastic strain energy of the different perturbed nanoring structures. Section 2 considers the general case of a surface traction, expressed in polar co-ordinates, that is distributed over an isotropic elastic half-space. This analysis is expanded to second order terms in relation to the radial slope of the initial ring to account for large slope effects. In section 3, a particular geometry for the nanoring is assumed, and an expression for the growth rate of different modal pertubations derived. The predictions of the model are presented in relation to experimental observations in section 4.

\section{Second order elastic strain energy of a generalised distribution of surface traction on an elastic half-space in polar coordinates}

The following analysis is applicable to any axisymmetric or non-axisymmetric heteroepitaxial nanostructure whose height profile, $\xi(r, \phi)=h(r) g(\phi)$, is readily expressed in terms of separate functions of the polar coordinates, $r$ and $\phi$, where $g(\phi)$ is represented by a Fourier series. Here we assume that the initial symmetric shape, defined by the radial height profile $h(r)$, is subjected to a time-dependent asymmetric undulation in the hoop direction such that

$$
\xi(\boldsymbol{r}, t)=\xi(r, \phi, t)=h(r)(1+\eta(\mathrm{t}) \cos n \phi)
$$


where $n$ and $\eta(t)$ are the mode number and relative amplitude of the undulation. It is easy to show that the undulation conserves volume. The axisymmetric problem $(n=0)$ has been extensively studied by Shchukin et al (2004) and Gill (2009). These linear analyses employed the small slope assumption (for slopes less than 0.15 ), whereby only first order surface tractions were considered. In this paper, second order terms are also included. This is partly because the observed slopes in the nanorings are relatively large (up to 0.3 ), but also because there are two interacting slopes in this case, the large radial slope of the initial nanoring, and the smaller slope in the hoop direction induced by the sinusoidal perturbation.

\subsection{First and second order surface tractions}

On a free surface, the normal, $\sigma_{N}$, and in-plane, $\sigma_{S} \& \sigma_{T}$, surface tractions should be zero. In 3D polar co-ordinates $(r, \phi, z)$ the out-of-plane slopes are given by

$$
\boldsymbol{s}(\boldsymbol{r})=\left[s_{r}, s_{\phi}\right]=\left[\frac{\partial \xi}{\partial r}, \frac{1}{r} \frac{\partial \xi}{\partial \phi}\right]
$$

where the components are in the radial and hoop directions respectively. The orientation of the surface defined by (1) is given by the corresponding radial, hoop and normal unit vectors

$$
\boldsymbol{S}=\frac{\left[1,0, s_{r}\right]}{\sqrt{1+s_{r}^{2}}}, \quad \boldsymbol{T}=\frac{\left[0,1, s_{\phi}\right]}{\sqrt{1+s_{\phi}^{2}}} \quad, \quad \boldsymbol{N}=\boldsymbol{S} \times \boldsymbol{T} .
$$

To second order in the slopes these are

$$
\boldsymbol{S}=\left[1-\frac{1}{2} s_{r}^{2}, 0, s_{r}\right], \quad \boldsymbol{T}=\left[0,1-\frac{1}{2} s_{\phi}^{2}, s_{\phi}\right], \quad \boldsymbol{N}=\left[-s_{r},-s_{\phi}, 1-\frac{1}{2}\left(s_{r}^{2}+s_{\phi}^{2}\right)\right] .
$$

The surface tractions are

$$
\sigma_{N}=N_{k} \sigma_{k l} N_{l} \quad \sigma_{S}=S_{k} \sigma_{k l} N_{l} \quad \sigma_{T}=T_{k} \sigma_{k l} N_{l}
$$

where $\sigma_{k l}$ is the stress tensor at the surface, and $(k, l)=(r, \phi, z)$. We expand the stress tensor into zeroth, first and second order terms

$$
\sigma_{k l}=\sigma_{k l}^{(0)}+\sigma_{k l}^{(1)}+\sigma_{k l}^{(2)}
$$

where the zeroth tensor is zero except for terms arising from the in-plane mismatch stress, $\sigma_{r r}^{(0)}=\sigma_{\phi \phi}^{(0)}=\sigma_{0}$, where $\sigma_{0}=\frac{E \epsilon_{0}}{1-v}, E$ and $v$ are the Young's modulus and Poisson ratio of the nanostructure and substrate (assumed to be the same) and $\epsilon_{0}$ is the mismatch strain.

Setting the surface stresses (5) to be zero gives to first order

$$
\sigma_{z z}^{(1)}=0 \quad \sigma_{i z}^{(1)}=\sigma_{0} s_{i}
$$

and to second order

$$
\sigma_{z z}^{(2)}=\sigma_{0}\left(s_{r}^{2}+s_{\phi}^{2}\right) \quad \sigma_{i z}^{(2)}=\sigma_{i j}^{(1)} s_{j}
$$

where $(i, j)=(r, \phi)$, in agreement with Pang and Huang (2006). Note that in (8) the first order surface stress components $\sigma_{r r}^{(1)}, \sigma_{r \phi}^{(1)}$ and $\sigma_{\phi \phi}^{(1)}$ are derived from the first order displacement field 
due to the first order surface tractions (7). Hence the first order problem must be solved to determine the boundary conditions for the second order correction.

\subsection{Determination of the first order elastic displacement}

Changes in the height of a strained nanostructure result in the generation of surface tractions on the substrate to balance the induced surface stresses (Gao, 1994). Following (6), we express the elastic displacement and applied surface tractions as the sum of first order and second order terms, such that $u_{i}=u_{i}^{(1)}+u_{i}^{(2)}$ and $f_{i}=f_{i}^{(1)}+f_{i}^{(2)}$. The first order elastic displacement $u_{i}^{(1)}$ is generated from the first order surface tractions $f_{i}^{(1)}$. Equation (7) shows that these tractions are confined to the surface plane such that

$$
f_{i}^{(1)}(\boldsymbol{r})=-\sigma_{i z}^{(1)}=-\sigma_{0} s_{i}
$$

The order $M$ contribution to the elastic displacement at a point $\boldsymbol{r}$ due to an in-plane surface traction distribution is given by

$$
u_{i}^{(M)}(\boldsymbol{r})=\int_{A} G_{i j}\left(\boldsymbol{r}-\boldsymbol{r}^{\prime}\right) f_{j}^{(M)}\left(\boldsymbol{r}^{\prime}\right) d^{2} \boldsymbol{r}^{\prime}
$$

where $A$ is the area of the surface under traction, and

$$
G_{i j}\left(\boldsymbol{r}-\boldsymbol{r}^{\prime}\right)=\frac{(1+v)}{\pi E}\left[\frac{(1-v)}{\left|\boldsymbol{r}-\boldsymbol{r}^{\prime}\right|} \delta_{i j}+\frac{v\left(\boldsymbol{r}-\boldsymbol{r}^{\prime}\right)_{i}\left(\boldsymbol{r}-\boldsymbol{r}^{\prime}\right)_{j}}{\left|\boldsymbol{r}-\boldsymbol{r}^{\prime}\right|^{3}}\right]
$$

is the Green's tensor (Landau and Lifshitz, 1970).

Following Shchukin et al. (2004) we use the following series expansions

$$
\begin{aligned}
& \frac{1}{\left|\boldsymbol{r}-\boldsymbol{r}^{\prime}\right|}=\sum_{m=0}^{\infty}\left(1-\frac{1}{2} \delta_{m 0}\right) \mathrm{p}_{\mathrm{m}}\left(\mathrm{r}_{<}, \mathrm{r}_{>}\right) \cos \left(m\left(\phi-\phi^{\prime}\right)\right) \\
& \frac{1}{\left|\boldsymbol{r}-\boldsymbol{r}^{\prime}\right|^{3}}=\sum_{m=0}^{\infty}\left(1-\frac{1}{2} \delta_{m 0}\right) \mathrm{q}_{\mathrm{m}}\left(\mathrm{r}_{<}, \mathrm{r}_{>}\right) \cos \left(m\left(\phi-\phi^{\prime}\right)\right)
\end{aligned}
$$

where $r_{<}=\min \left(r, r^{\prime}\right)$ and $r_{>}=\max \left(r, r^{\prime}\right)$, and the radial functions are

$$
\begin{aligned}
& p_{m}\left(r_{<}, r_{>}\right)=\frac{2}{\pi r_{>}} \sum_{p=0}^{\infty} \frac{\Gamma\left(|m|+\frac{1}{2}+p\right) \Gamma\left(\frac{1}{2}+p\right)}{\Gamma(|m|+1+p) \Gamma(1+p)}\left(\frac{r_{\leq}}{r_{>}}\right)^{|m|+2 p} \\
& q_{m}\left(r_{<}, r_{>}\right)=\frac{8}{\pi r_{>}^{3}} \sum_{p=0}^{\infty} \frac{\Gamma\left(|m|+\frac{3}{2}+p\right) \Gamma\left(\frac{3}{2}+p\right)}{\Gamma(|m|+1+p) \Gamma(1+p)}\left(\frac{r_{\leq}}{r_{>}}\right)^{|m|+2 p}
\end{aligned}
$$

where $\Gamma(p)$ is the Gamma function. In practise a finite number of terms in the series, $N$, are used. Note that if $m<0$ then one less term in the finite series expansion must be taken, i.e. $(N-1)$. This is because the final solution arises from the accumulation of small differences between large terms in different series and hence the terms have to be paired correctly to avoid significant errors. This is only needed for the evaluation of $q_{n-2}$ for the situation where $n=1$.

Substituting (9), (11) and (12) into (10) gives

$$
u_{i}^{(1)}(r, \phi)=-\frac{(1+v) \epsilon_{0}}{(1-v) \pi} \int_{R-a}^{R+a}\left((1-v) P_{i}+v Q_{i}\right) r^{\prime} d r^{\prime}
$$


where

$$
\begin{aligned}
& P_{i}\left(r, \phi, r^{\prime}\right)=\sum_{m=0}^{\infty}\left(1-\frac{1}{2} \delta_{m 0}\right) p_{m}\left(r_{<}, r_{>}\right) \int_{0}^{2 \pi} \cos \left(m\left(\phi-\phi^{\prime}\right)\right) s_{i}\left(\boldsymbol{r}^{\prime}\right) d \phi^{\prime} \\
& Q_{i}\left(r, \phi, r^{\prime}\right)=\sum_{m=0}^{\infty}\left(1-\frac{1}{2} \delta_{m 0}\right) q_{m}\left(r_{<}, r_{>}\right) \int_{0}^{2 \pi}\left(\boldsymbol{r}-\boldsymbol{r}^{\prime}\right)_{i}\left(\boldsymbol{r}-\boldsymbol{r}^{\prime}\right)_{j} \cos \left(m\left(\phi-\phi^{\prime}\right)\right) s_{j}\left(\boldsymbol{r}^{\prime}\right) d \phi^{\prime}
\end{aligned}
$$

The radial vector $\boldsymbol{r}=[r, 0]$ is chosen to define the polar coordinate system for the integration over $d^{2} \boldsymbol{r}^{\prime}$ such that

$$
\boldsymbol{r}-\boldsymbol{r}^{\prime}=\left[r-r^{\prime} \cos \left(\phi-\phi^{\prime}\right), r^{\prime} \sin \left(\phi-\phi^{\prime}\right)\right]
$$

and

$$
\boldsymbol{s}\left(\boldsymbol{r}^{\prime}\right)=\left[s_{r}^{\prime} \cos \left(\phi-\phi^{\prime}\right)+s_{\phi}^{\prime} \sin \left(\phi-\phi^{\prime}\right),-s_{r}^{\prime} \sin \left(\phi-\phi^{\prime}\right)+s_{\phi}^{\prime} \cos \left(\phi-\phi^{\prime}\right)\right]
$$

where, from (2), $s_{r}^{\prime}=\partial \xi\left(r^{\prime}, \phi^{\prime}\right) / \partial r^{\prime}$ etc.

Given (1) the integrals (15a) can be readily evaluated to yield

$$
\begin{aligned}
& P_{r}\left(r, \phi, r^{\prime}\right)=\pi p_{1} \frac{d h^{\prime}}{d r^{\prime}}+\frac{1}{2} \eta \pi\left[\frac{d h^{\prime}}{d r^{\prime}}\left(p_{n+1}+p_{n-1}\right)-\frac{n h^{\prime}}{r^{\prime}}\left(p_{n+1}-p_{n-1}\right)\right] \cos n \phi \\
& P_{\phi}\left(r, \phi, r^{\prime}\right)=\frac{1}{2} \eta \pi\left[\frac{d h^{\prime}}{d r^{\prime}}\left(p_{n+1}-p_{n-1}\right)-\frac{n h^{\prime}}{r^{\prime}}\left(p_{n+1}+p_{n-1}\right)\right] \sin n \phi
\end{aligned}
$$

and (15b) become

$$
\begin{gathered}
Q_{r}\left(r, \phi, r^{\prime}\right)=\frac{1}{2} \eta \pi\left[\begin{array}{c}
\frac{d h^{\prime}}{d r^{\prime}}\left[\left(r^{2}+r^{\prime 2}\right)\left(q_{n+1}+q_{n-1}\right)-r r^{\prime}\left(3 q_{n}+\frac{1}{2}\left(q_{n+2}+q_{n-2}\right)\right)\right] \\
-n h^{\prime}\left[r\left(q_{n+1}-q_{n-1}\right)-\frac{1}{2} r^{\prime}\left(q_{n+2}-q_{n-2}\right)\right]
\end{array}\right] \cos n \phi \\
Q_{\phi}\left(r, \phi, r^{\prime}\right)=\frac{1}{2} \eta \pi\left[\begin{array}{c}
\frac{d h^{\prime}}{d r^{\prime}}\left[\begin{array}{c}
\left.r^{\prime 2}\left(q_{n+1}-q_{n-1}\right)-\frac{1}{2} r r^{\prime}\left(q_{n+2}-q_{n-2}\right)\right] \\
-n h^{\prime} r\left[q_{n}-\frac{1}{2}\left(q_{n+2}+q_{n-2}\right)\right]
\end{array}\right] \sin n \phi
\end{array}\right]
\end{gathered}
$$

where $h^{\prime}=h\left(r^{\prime}\right)$, and $p_{m}$ and $q_{m}$ are functions of $r$ and $r^{\prime}$. The following relation has been employed

$$
\left(r^{2}+r^{\prime 2}\right) q_{1}=r r^{\prime}\left(3 q_{0}+\frac{1}{2} q_{2}\right)
$$

This renders the contribution to the $Q$ terms for purely axisymmetric problems zero. Note that only the first term in (18a) arises from the initial ring structure. All the other $(\eta)$ terms arise from the nonaxisymmetric sinusoidal perturbation.

We now introduce the following dimensional quantities to describe the initial nanoring. For a ring of inner diameter $d_{i}$ and outer diameter $d_{o}$, we define the shape in terms of the mid-radius, $R=\frac{1}{4}\left(d_{o}+d_{i}\right)$, the half thickness, $a=\frac{1}{4}\left(d_{o}-d_{i}\right)$, and the peak height, $h_{o}$. The size of the ring is defined by $R$ and the shape is defined by the dimensionless quantities $s_{0}=\frac{h_{0}}{a}$, the average radial slope, and $\beta=\frac{a}{R}$, the relative ring thickness. Let $\bar{r}=r / R$ and $\bar{h}=\frac{h}{h_{0}}$ such that we can have $\bar{u}=$ 
$\frac{u}{R^{\prime}}, \bar{p}_{m}=R p_{m}, \bar{q}_{m}=R^{3} q_{m}$ and consequently $\bar{P}_{i}=\left(\frac{R}{h_{0}}\right) R P_{i}=\left(s_{0} \beta\right)^{-1} R P_{i}$ and $\bar{Q}_{i}=\left(\frac{1}{h_{0} R}\right) R^{3} Q_{i}=$ $\left(s_{0} \beta\right)^{-1} R Q_{i}$. Thus the elastic displacement (14) can finally be written as

$$
\bar{u}_{i}^{(1)}(\bar{r}, \phi)=-\frac{(1+v) \epsilon_{0}}{(1-v)} s_{0} \beta \bar{U}_{i}(\bar{r}, \phi ; v, \beta)
$$

where the function

$$
\bar{U}_{i}(\bar{r}, \phi ; v, \beta)=\frac{1}{\pi} \int_{1-\beta}^{1+\beta}\left[(1-v) \bar{P}_{i}\left(\bar{r}, \phi, \bar{r}^{\prime}\right)+v \bar{Q}_{i}\left(\bar{r}, \phi, \bar{r}^{\prime}\right)\right] \bar{r}^{\prime} d \bar{r}^{\prime}
$$

If the radial profile of the ring, $\bar{h}(\bar{r})$, is a polynomial, then the $\bar{P}_{i}$ and $\bar{Q}_{i}$ functions are also simple polynomial series. The above integrals are therefore analytically trivial to compute. However, due to the complexity of the resulting algebra, this task was undertaken computationally using the mathematical package Maple. The first 250 terms in the series are considered to achieve good convergence. Importantly, the numerical accuracy of the calculation must be increased (to 20 digits) to ensure proper convergence. The resulting expression for the displacements are of the form $\bar{U}_{r}(\bar{r}, \phi)=\bar{U}_{r 0}(\bar{r})+\eta \bar{U}_{r n}(\bar{r}) \cos (n \phi)$ and $\bar{U}_{\phi}(\bar{r}, \phi)=\eta \bar{U}_{\phi n}(\bar{r}) \sin (n \phi)$, where the various functions are polynomial series in $\bar{r}$ plus a finite $\bar{r} \ln (\bar{r})$ term.

\subsection{Determination of the first and second order elastic strain energy}

The change in the elastic strain energy from the initially flat configuration due to a general surface traction distribution is given by

$$
\Delta \mathrm{E}_{\mathrm{e}}=-\frac{1}{2} \int_{A} u_{i}(\boldsymbol{r}) f_{i}(\boldsymbol{r}) d^{2} \boldsymbol{r}
$$

Now express the strain energy as the sum of first and second order components such that $\Delta E_{e}=\Delta E_{e}^{(1)}+\Delta E_{e}^{(2)}$. We can therefore write

$$
\Delta \mathrm{E}_{\mathrm{e}}^{(1)}=-\frac{1}{2} \int_{R-a}^{R+a} \int_{0}^{2 \pi} u_{i}^{(1)} f_{i}^{(1)} r d \phi d r
$$

and

$$
\Delta \mathrm{E}_{\mathrm{e}}^{(2)}=-\frac{1}{2} \int_{R-a}^{R+a} \int_{0}^{2 \pi}\left(u_{i}^{(1)} f_{i}^{(2)}+u_{i}^{(2)} f_{i}^{(1)}\right) r d \phi d r .
$$

Now we can make use of the identity

$$
\begin{aligned}
\int_{A} u_{i}^{(2)} f_{i}^{(1)} d^{2} \boldsymbol{r} & =\int_{A} \int_{A} f_{i}^{(1)}(\boldsymbol{r}) G_{i j}\left(\boldsymbol{r}-\boldsymbol{r}^{\prime}\right) f_{j}^{(2)}\left(\boldsymbol{r}^{\prime}\right) d^{2} \boldsymbol{r}^{\prime} d^{2} \boldsymbol{r} \\
& =\int_{A} \int_{A} f_{i}^{(2)}(\boldsymbol{r}) G_{i j}\left(\boldsymbol{r}-\boldsymbol{r}^{\prime}\right) f_{j}^{(1)}\left(\boldsymbol{r}^{\prime}\right) d^{2} \boldsymbol{r}^{\prime} d^{2} \boldsymbol{r}=\int_{A} u_{i}^{(1)} f_{i}^{(2)} d^{2} \boldsymbol{r}
\end{aligned}
$$

to write (25) as

$$
\Delta \mathrm{E}_{\mathrm{e}}^{(2)}=-\int_{R-a}^{R+a} \int_{0}^{2 \pi} u_{i}^{(1)} f_{i}^{(2)} r d \phi d r
$$

This avoids explicit determination of $u_{i}^{(2)}$ which is expensive. The applied second order in-plane surface tractions are given by (8)

$$
f_{i}^{(2)}=-\sigma_{i j}^{(1)} s_{j}
$$


There is an out-of-plane second order surface traction $f_{z}^{(2)}$ but $u_{z}^{(1)}$ is zero everywhere so the product of these makes no contribution to the second order strain energy (27). Note that similarly $u_{z}^{(2)}$ is non-zero but, from (7), the out-of-plane first order surface traction $f_{z}^{(1)}$ is zero so the product of these two terms would also make no contribution, in accordance with the identity (26). It is useful at this point to re-adopt the non-dimensional form of the previous subsection. The surface strains are related to the surface displacements by the usual relationships such that

$$
\epsilon_{i j}=-\frac{(1+v) \epsilon_{0}}{(1-v)} s_{0} \beta \bar{\epsilon}_{i j}
$$

where

$$
\bar{\epsilon}_{r r}=\frac{\partial \bar{U}_{r}}{\partial \bar{r}} \quad \bar{\epsilon}_{\phi \phi}=\frac{\bar{U}_{r}}{\bar{r}}+\frac{1}{\bar{r}} \frac{\partial \bar{U}_{\phi}}{\partial \phi} \quad \bar{\gamma}_{r \phi}=\frac{1}{\bar{r}} \frac{\partial \bar{U}_{r}}{\partial \phi}+\frac{\partial \bar{U}_{\phi}}{\partial \bar{r}}-\frac{\bar{U}_{\phi}}{\bar{r}} .
$$

The surface stresses are then

$$
\sigma_{i j}=-\frac{E \epsilon_{0}}{(1-v)^{2}} s_{0} \beta \bar{\sigma}_{i j}
$$

where

$$
\bar{\sigma}_{r r}=\bar{\epsilon}_{r r}+v \bar{\epsilon}_{\phi \phi} \quad \bar{\sigma}_{\phi \phi}=\bar{\epsilon}_{\phi \phi}+v \bar{\epsilon}_{r r} \quad \bar{\sigma}_{r \phi}=\frac{1}{2}(1-v) \bar{\gamma}_{r \phi}
$$

Expressing the slopes in dimensionless form as $\bar{s}_{i}=\left(\frac{R}{h_{0}}\right) s_{i}=\left(s_{0} \beta\right)^{-1} s_{i}$, we can now write the first and second order contributions to the change in total strain energy from (24) and (27) as

$$
\begin{aligned}
\Delta \overline{\mathrm{E}}_{\mathrm{e}}^{(1)} & =-\frac{1}{4 \pi} \int_{1-\beta}^{1+\beta} \int_{0}^{2 \pi} \bar{U}_{i} \bar{s}_{i} \bar{r} d \phi d \bar{r} \\
\Delta \overline{\mathrm{E}}_{\mathrm{e}}^{(2)} & =\frac{1}{2 \pi(1-v)} \int_{1-\beta}^{1+\beta} \int_{0}^{2 \pi} \bar{U}_{i} \bar{\sigma}_{i j} \bar{s}_{j} \bar{r} d \phi d \bar{r}
\end{aligned}
$$

where $\Delta \bar{E}_{e}=\frac{\Delta E_{e}}{E_{e}}$ is written as $\Delta \bar{E}_{e}=\Delta \bar{E}_{e}^{(1)}+\left(\mathrm{s}_{0} \beta\right) \Delta \bar{E}_{e}^{(2)}$ to explicitly show the slope dependence of the second order term. The reference strain energy is $E_{e}=2 \pi \frac{(1+v)}{(1-v)} w_{0} R^{3}\left(s_{0} \beta\right)^{2}$ and the strain energy density of the reference surface is $w_{0}=\sigma_{0} \epsilon_{0}=\frac{E \epsilon_{0}^{2}}{(1-v)}$. The second order term only becomes significant as the radial slope of the initial nanoring, $s_{0}$, becomes large.

It is possible to express the total change in strain energy up to second order as the sum of (33a) and (33b) in the form

$$
\Delta \bar{E}_{e}=-\left(\Delta \bar{E}_{e 0}+\eta^{2} \Delta \bar{E}_{e n}\right)
$$

where $\Delta \bar{E}_{e 0}=\Delta \bar{E}_{e 0}^{(1)}+\left(\mathrm{s}_{0} \beta\right) \Delta \bar{E}_{e 0}^{(2)}$ is the mode 0 term (strain relaxation energy due to the formation of the initial nanoring), and $\Delta \bar{E}_{e n}=\Delta \bar{E}_{e n}^{(1)}+\left(\mathrm{s}_{0} \beta\right) \Delta \bar{E}_{e n}^{(2)}$ is the mode $n$ term due to changes in strain energy due to the sinusoidal undulation. Both terms have first and second order contributions. The dimensionless variable, $\eta(t)$, represents the amplitude of the undulation and is shown explicitly in (34) as it is the only time-dependent quantity. An evaluation of the second order elasticity model defined by (33) is given in Appendix A in the context of the conical island of Shchukin 
et al (2004). The first order elastic strain energy (33a) was originally derived by a different method using Fourier transforms (see Supplementary Material). It is a useful confirmation of (33a) that both methods produce the same numerical results.

\section{Linear stability analysis of a nanoring}

\subsection{Geometrical assumptions}

The simplest expression for a continuous radial height profile is a quadratic polynomial. Assuming that the peak height occurs at $r=R$ gives

$$
h(r)=h_{0}\left(1-\frac{(R-r)^{2}}{a^{2}}\right) .
$$

Examples of representative nanorings described by (1) and (35) are shown in Fig.1.

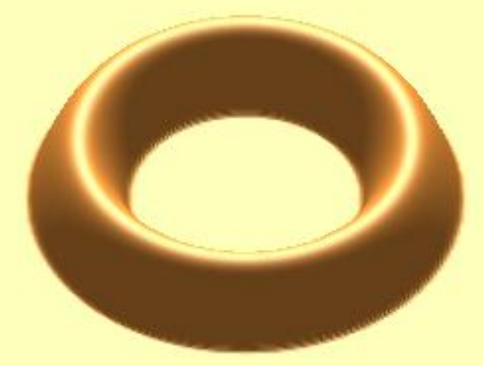

(a)

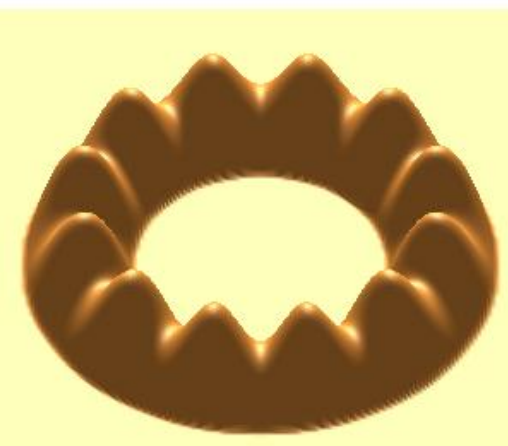

(b) 


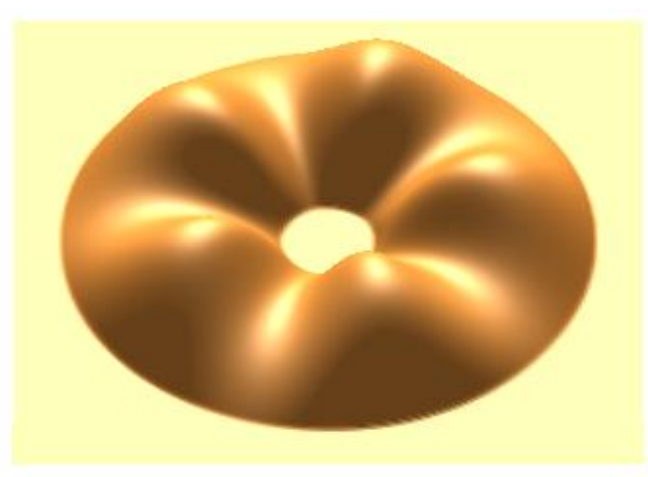

(c)

Fig. 1. Examples of nanoring geometries : (a) an initial nanoring with $\eta=0$ and $a=0.3 R$, (b) an undulating nanoring with $a=0.3 R$ and $n=12$, (c) an undulating nanoring with $a=0.7 R$ and $n=5$.

The volume of the nanoring is

$$
V=\int_{R-a}^{R+a} \int_{0}^{2 \pi} \xi(r, \phi) r d \phi d r=\frac{8 \pi}{3} R a h_{0}
$$

The surface area of the nanoring is

$$
A_{s}=\int_{R-a}^{R+a} \int_{0}^{2 \pi} \sqrt{1+\left(\frac{d \xi}{d r}\right)^{2}+\left(\frac{1}{r} \frac{d \xi}{d \phi}\right)^{2}} r d \phi d r .
$$

This can be expanded up to second order as $A_{S}=A_{S}^{(0)}+A_{S}^{(1)}+A_{S}^{(2)}$, where $A_{S}^{(0)}=4 \pi R a$,

$$
A_{S}^{(1)}=\frac{8 \pi R h_{0}^{2}}{3 a}+\eta^{2} \pi h_{0}^{2}\left[\frac{4 R}{3 a}+n^{2}\left(\frac{10 R}{3 a}-\frac{2 R^{3}}{a^{3}}+\left(\frac{R^{2}}{a^{2}}-1\right)^{2} \ln \left(\frac{R+a}{R-a}\right)\right)\right] .
$$

and $A_{S}^{(2)}=0$. The zeroth order term is the basal area of the ring, so the change in surface energy upon nanoring formation is

$$
\Delta E_{S}=\gamma_{1} A_{S}-\gamma_{0} A_{S}^{(0)}
$$

where $\gamma_{0}$ is the surface energy density of the substrate and $\gamma_{1}$ is that of the nanoring. This can then be expressed as decoupled mode 0 and mode $n$ components

$$
\Delta E_{s}=\Delta E_{s 0}+\eta^{2} \Delta E_{s n}
$$

Hence the perturbation to the surface energy, calculated from (38) and (39), can be written in dimensionless form as

$$
\Delta \bar{E}_{s n}(n, \beta)=\left[\frac{4}{3}+n^{2}\left(\frac{10}{3}-\frac{2}{\beta^{2}}+\beta\left(\frac{1}{\beta^{2}}-1\right)^{2} \ln \left(\frac{1+\beta}{1-\beta}\right)\right)\right]
$$

where $\Delta \bar{E}_{s n}=\Delta E_{s n} / E_{S}$ and the reference surface energy is $E_{S}=\gamma_{1} \pi s_{o}^{2} \beta R^{2}$.

\subsection{Growth rate of the perturbation}


Any pre-existing undulating perturbation to the nanoring shape is assumed to evolve via surface diffusion. The evolution of the nanoring can therefore be defined by the variational functional (Cocks et al, 1999; Suo, 1995)

$$
\Pi=\int_{A_{S}} \frac{j_{s}^{2}}{2 D_{S}} d A+\Delta \dot{E}_{e}+\Delta \dot{E}_{S}
$$

where the first term is the dissipation potential, $D_{S}$ is the constant surface diffusivity and $j_{S}$ is the surface flux. The last two terms are the driving forces for the evolution, due to changes in the elastic and surface energy of the system, where the dot denotes differentiation with respect to time. It is expected that the surface energy will have a stabilising influence, and that the elastic strain energy will always act to destabilise the system. For the assumed surface profile (1) the flux of atoms over the surface must be purely in the hoop direction, such that

$$
\frac{1}{r} \frac{d j_{s}}{d \phi}+v_{N}=0
$$

where the normal velocity of the surface $v_{N} \approx \dot{\xi}$. Consequently

$$
j_{s}=-\dot{\eta} \int_{0}^{\phi} r h(r) \cos (n \phi) d \phi=-\frac{\dot{\eta} r h(r) \sin (n \phi)}{n},
$$

and the dissipation potential in (42) is

$$
\int_{R-a}^{R+a} \int_{0}^{2 \pi} \frac{j_{s}^{2}}{2 D_{s}} r d \phi d r=\frac{8 \pi \dot{\eta}^{2} h_{0}^{2} R a\left(7 R^{2}+3 a^{2}\right)}{105 D_{s} n^{2}}
$$

The variational function (42) is therefore given by (34), (41) and (45) to be

$$
\Pi=\frac{8 \pi \dot{\eta}^{2} h_{0}^{2} R a\left(7 R^{2}+3 a^{2}\right)}{105 D_{s} n^{2}}+2 \eta \dot{\eta}\left(\Delta E_{s n}-\Delta E_{e n}\right) .
$$

The actual evolution path of the system is that which minimizes the variational functional with respect to the kinetic degree-of-freedom, i.e. when $\frac{d \Pi}{d \dot{\eta}}=0$. Hence (46) yields

$$
\dot{\eta}=\alpha \eta
$$

where the growth exponent

$$
\alpha=\frac{105 D_{S} n^{2}\left(\Delta E_{e n}-\Delta E_{S n}\right)}{8 \pi h_{0}^{2} R a\left(7 R^{2}+3 a^{2}\right)} .
$$

From (47), the growth of the perturbation is given by $\eta(t)=\eta(0) \exp (\alpha t)$, such that perturbations with $\alpha>0$ will grow, whereas those with $\alpha<0$ will decay. It is expected that the perturbation that is physically observed will be the one that maximises the value of the growth exponent. Note that the contributions from the elastic strain energy, $\Delta E_{e n}$, and surface energy, $\Delta E_{s n}$, are complicated functions of the initial ring geometry $\left(s_{0}\right.$ and $\left.\beta\right)$ and the mode number $(n)$.

To reduce the number of system parameters, the growth rate (48) is expressed in dimensionless form

$$
\bar{\alpha}\left(n ; s_{0}, \beta, R ; v, L_{0}\right)=\frac{n^{2}}{\beta^{2}\left(7+3 \beta^{2}\right)}\left(\frac{L_{0}}{R}\right)^{3}\left(\beta \bar{E}_{e n}\left(n, s_{0}, \beta ; v\right)-\left(\frac{L_{0}}{R}\right) \bar{E}_{s n}(n, \beta)\right),
$$


where $\bar{\alpha}=\alpha \tau_{0}$, and

$$
\tau_{0}=\frac{8 L_{0}^{4}}{105 D_{s} \gamma_{1}}
$$

is a fixed characteristic time, and

$$
L_{0}=\frac{(1-v) \gamma_{1}}{2(1+v) w_{0}}
$$

is a material length scale which arises from the relative competition between the surface and strain energies.

\section{Results}

The growth rate of the perturbation is a function of the mode number $n$, the geometry of the ring (defined by the ring radius $R$, the half-width to radius ratio, $\beta=\frac{a}{R}$, and the mean radial slope, $s_{0}=\frac{h_{0}}{a}$ ), and the material properties of the system (represented by Poisson ratio, $v$, and material length scale $L_{0}$ ). The effect of changing the Poisson ratio over the physically significant range (0.20.4 ) is small and hence for the purposes of this study we assume the Poisson ratio of InP which is $\nu=0.36$. Firstly we explore the parameter space within the context of linear, small slope theory (effectively setting $s_{0}=0$ ), investigating how the volume of a nanoring affects its stability for different shapes. The effects of the non-linear, second order elastic terms (finite $s_{0}$ ) are then included in the subsequent section to determine the consequences for the evolution of larger sloped nanorings. Finally, the effect of changing the shape under constant volume conditions is evaluated.

\subsection{Variable volume}

\section{(a) first order (small slope) analysis}

The experiments of Jevasuwan et al. (2013) determine the consequences of varying the nanoring volume by changing the quantity of material that is deposited. They find that after $1.6 \mathrm{ML}$ of InP have been deposited, at a deposition temperature of $200^{\circ} \mathrm{C}$ and a crystallisation temperature of $250^{\circ} \mathrm{C}$, the nanorings have a radius of approximately $40 \mathrm{~nm}$ and that they are stable, i.e. no quantum dot molecules (QDMs) are formed. Increasing the amount of material deposited to 3.2ML sees an increase in the average radius to about $75 \mathrm{~nm}$ and a destabilisation of the nanorings, such that QDMs consisting of 8-12 quantum dots are observed. Further increases in deposition to $4.8 \mathrm{ML}$ and $6.4 \mathrm{ML}$ see a further increase in the ring size to about $100 \mathrm{~nm}$, and a small increase in the number of quantum dot molecules roughly in the range of 10-14. These observations are now explored in the context of the stability model (49).

Measurement of the nanorings and quantum dot molecules shows that the half-width to radius ratio does not change significantly between experiments, with $\beta=0.25-0.3$. The height-to-width ratio

also remains reasonably constant with $s_{0}=\frac{h_{0}}{a}=0.1-0.15$. For the assumed profile (35) the maximum slope $2 s_{0}$ occurs at the perimeters. This exceeds the valid limits of first order analysis, for which linearity is generally assumed to only hold for slopes up to about 0.1-0.15 (see Appendix A). 
However, the general behaviour of the system is initially explored here in the context of first order theory. The effect of second order corrections are considered in the next section.

The mismatch strain in the $\operatorname{InP}$ on $\operatorname{In}_{0.5} \mathrm{Ga}_{0.5} \mathrm{P}$ system is $\epsilon_{0}=-3.8 \%$, and the modulus and Poisson ratio of $\operatorname{InP}$ are $E=61.1 \mathrm{GPa}$ and $v=0.36$. The surface energies of $\mathrm{InP}$ vary between $0.5-1.5 \mathrm{~J} / \mathrm{m}^{2}$ depending on orientation and strain (Liu et al, 1999). The best correlation between experiment and theory is obtained for $\gamma_{1}=1.5 \mathrm{~J} / \mathrm{m}^{2}$ so this is the assumed value, although it is recognised that the idealised assumptions of the model are not expected to exactly reproduce the experimental observations, but should indicate the principle factors and trends of the system with some quantitative accuracy. The above material data defines the material length scale to be $L_{0}=2.6 \mathrm{~nm}$ for InP on $\ln _{0.5} \mathrm{Ga}_{0.5} \mathrm{P}$.

Fig. 2 shows the growth rates for different mode numbers $n$ as a function of the ring geometry, $\beta$, for a value of $R / L_{0}=30$. Only the modes with $n=1$ to 9 grow, so that modes with $n>9$ are stable. The growth rate is maximised for most values of $\beta$ for $n=6$ or 7 . The fastest growing mode is calculated from (49) and plotted in Fig. 3a for rings of different radius and width. It is clear that there are regions of the parameter space where nanorings are unconditionally stable. This suggests that nanorings with a small radius or a small width-to-radius ratio will not evolve into QDMs. A nanoring will become unstable once its radius increases beyond a certain limit. Beyond this limit the expected number of quantum dots $(n)$ in the resulting QDM increases with the radius. Once in the unstable region, the optimal mode shows little sensitivity to the width-to-radius ratio of the nanoring. The respective growth rates of the optimal modes are shown in Fig. 3b. The corrugations in the contours correspond to a discrete change in the modal number. Overall, there is not a hugely significant change in the growth rate of the fastest mode, except near the boundary with the stable region.

The experimental observations of Jevasuwan et al. (2013) are shown as three points in Fig. 3a. The nanoring corresponding to $1.6 \mathrm{ML}$ is found to be on the boundary of the stable region, which agrees with the finding that these nanorings do not break-up in to QDMs within the duration of the experiment. The larger nanorings formed when 3.2ML and 4.8ML are deposited are found to be well within the unstable region. The optimal mode number at these deposition thicknesses are $n=6-7$ and $n=8-9$ respectively. This is slightly below the observed ranges of 8-12 and 10-14, but the model certainly demonstrates the correct trend and is quantitatively very reasonable within the necessary assumptions of a simplified analysis.

Generally it can be seen in Fig. 3a that for reasonably thick rings, $0.3<\beta<0.7$, the mode number increases fairly uniformly with the relative ring radius, $R / L_{0}$. This is similar to the flat film case (Gill, 2003) for which the fastest growing wavelength, $\frac{4 \pi \gamma_{1}}{3(1+v) w_{0}}=\frac{8 \pi(1-v)}{3} L_{0}$, is equal to $5.4 L_{0}$ for $v=0.36$. Here the preferred wavelength of the perturbation is the circumference of the ring divided by the number of waves. It is found from Fig. 3 a that $\frac{2 \pi R}{n} \approx 28 L_{0}$ for $v=0.36$. This is considerably larger than the wavelength for the flat film, suggesting that the ring cannot elastically relax as readily. Hence rings with $R<4.5 L_{0}$ are stable as their circumference is not large enough to accommodate a single wave of this size, forming a rough lower (horizontal) bound to the stable region. The other (vertical) part of the stable zone, roughly described by $\beta<0.1$ in Fig. 3a, shows that rings with large radii and small thickness do not elastically relax as effectively as those with radii that are smaller 
relative to their thickness. The $\operatorname{InP}(1.6 \mathrm{ML})$ ring is on the transition region between these two contributions to the stability.

Another nanoring system, grown under heteroepitaxial conditions, that can be used for further comparison concerns the growth of InGaAs nanorings on GaAs by Hanke et al. (2007) by partial capping. This growth process necessarily leads to intermixing of the components, which renders precise comparison with theory difficult. However Hanke et al (2007) analyse the resulting nanorings in this system as uniformly alloyed $\ln _{0.4} \mathrm{Ga}_{0.6} \mathrm{As}$ and obtain reasonable agreement with experiment. The nanorings in this system are stable and no QDMs are observed. The typical dimensions of the rings are inner diameter $15 \mathrm{~nm}$, outer diameter $59 \mathrm{~nm}$ and height $0.8 \mathrm{~nm}$. The radius and half-width of the rings are therefore $R=18 \mathrm{~nm}$ and $a=11 \mathrm{~nm}$. Taking the elastic properties of this alloy system to be $E=71 \mathrm{GPa}, v=0.33$ and $\epsilon_{0}=-2.7 \%$, the estimated material length scale is $L_{0}=6.5 \mathrm{~nm}$ for a typical surface energy of $\gamma_{1}=1 \mathrm{~J} / \mathrm{m}^{2}$. The shape and size of these rings are therefore defined by $\beta=0.6$ and $R / L_{0} \approx 3$. This point for the InGaAs system, shown in Fig. 3a, is clearly within the lower stable regime, consistent with experimental observations.

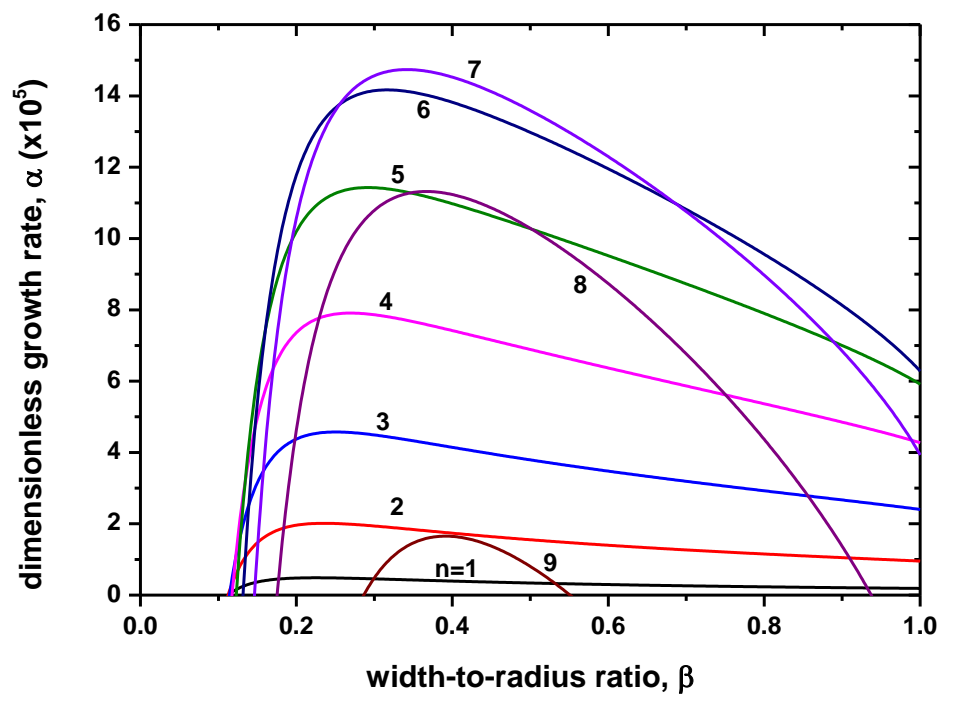

Fig 2 : Example of the growth rate $\bar{\alpha}$ of different modes as a function of $\beta$ for varying volume fors $s_{0}=0, R / L_{0}=30$ and $v=0.36$. Only the modes with $n=1$ to 9 grow. Modes with $n>9$ are stable. The growth rate is maximised for most shapes when $n=6$ or 7 . 


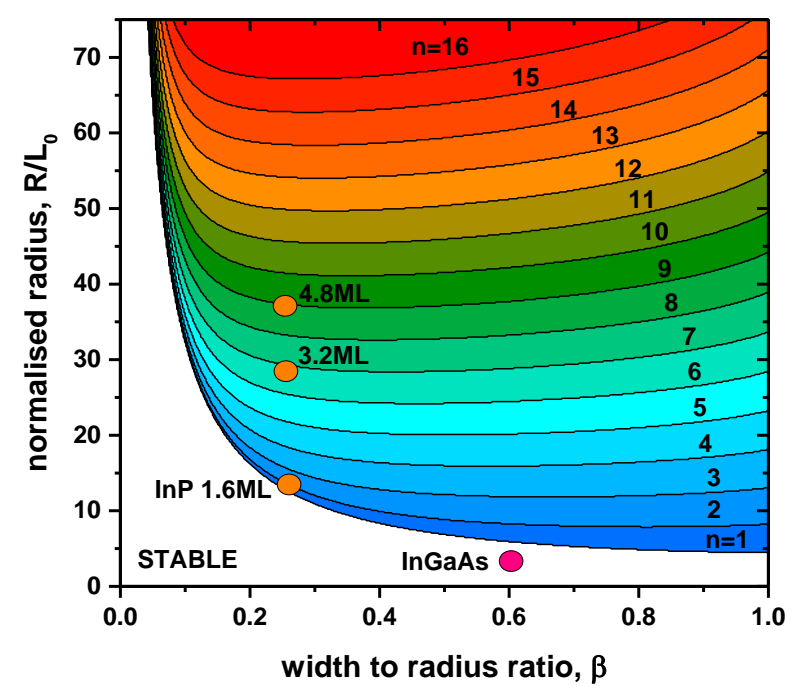

(a)

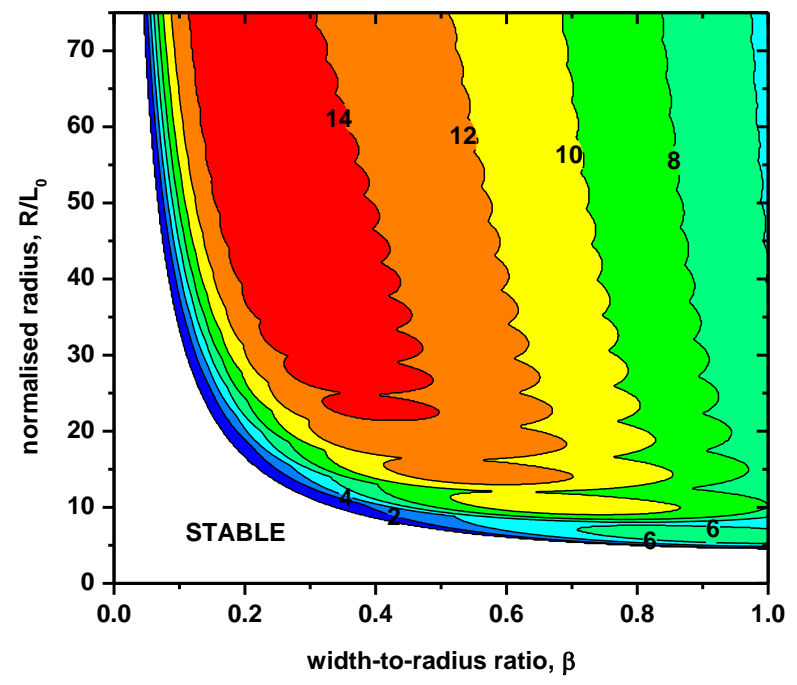

(b)

Fig. 3 : For small slopes (a) optimal mode number $n$ and (b) optimal growth rate $\bar{\alpha}\left(x 10^{5}\right)$ for rings with variable size, $R / L_{0}$, and shape, $\beta$ and $v=0.36$. The size and shape of different observed rings are shown as points on the stability map in (a). Three rings of different sizes are shown for the InP on $\mathrm{In}_{0.5} \mathrm{Ga}_{0.5} \mathrm{P}$ system $\left(L_{0}=2.6 \mathrm{~nm}\right)$ corresponding to different levels of deposition. After $1.6 \mathrm{ML}$ the rings are found to be stable, but after 3.2ML and above they are not. The $\ln _{0.4} \mathrm{Ga}_{0.6} \mathrm{As}$ on $\mathrm{GaAs}$ rings $\left(L_{0}=6.5 \mathrm{~nm}\right)$ are clearly in the stable region as observed in experiment.

(b) second order (finite slope) analysis

As discussed in the previous subsection, the mean radial slope for the InP nanorings is $s_{0}=0.1-$ 
0.15 with a peak slope of $2 s_{0}=0.2-0.3$ at the perimeter. These slopes are expected to be slightly beyond the range of validity for the first order analysis, and so the effect of finite slopes is now explored by adopting the full second order elasticity model of (34). Figs. $4 a$ and $4 b$ are similar to Fig. 3a except that $s_{0}=0.1$ and 0.3 in the former and $s_{0}=0$ in the latter. It can be seen in Fig. 4a that the contours have moved slightly upward compared to Fig. 3a. This shows that the elastic relaxation is marginally reduced in the finite slope case, such that the surface energy is more dominant and larger wavelengths prevail. The fairly extreme case of $s_{0}=0.3$ in Fig. $4 \mathrm{~b}$ shows that these effects are more considerable when very large slopes are considered. The experimentally observed nanoring structures are plotted in Fig. $4 \mathrm{a}$ as in Fig. 3a. According to the measurements, the model predictions in Fig. 4a should more closely reflect the actual situation. The differences between the two are small, although the region of stability in Fig. 4a has increased, to place the $\operatorname{InP}(1.6 \mathrm{ML})$ nanoring more clearly in the stable zone, as expected. Fig. 5 summarises the effect of a finite slope on the stability of the system. The boundary lines between the stable $(n=0)$ and unstable $(n>0)$ regions are shown for different values of the mean radial slope. As the slope increases, the elastic relaxation energy decreases, and the region of stability expands.

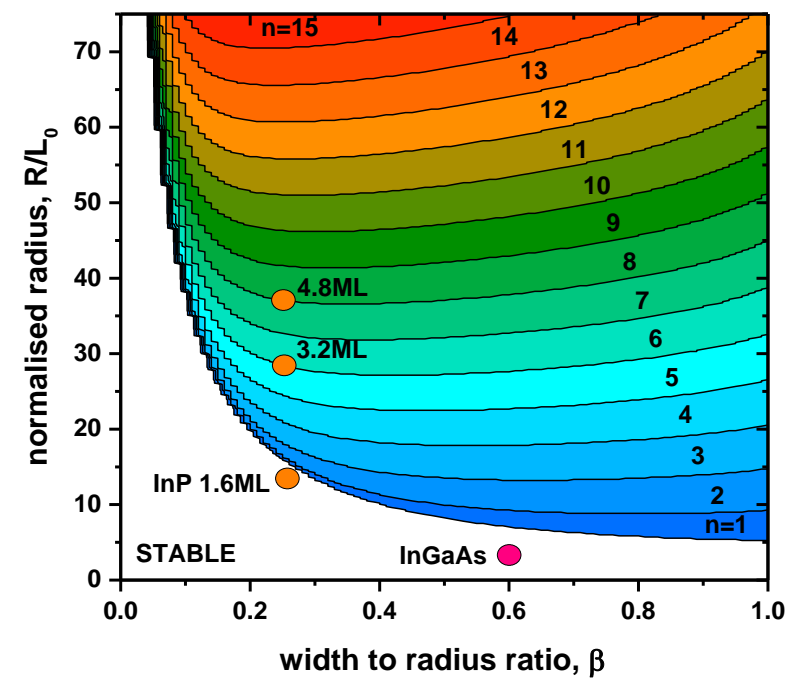

(a) 


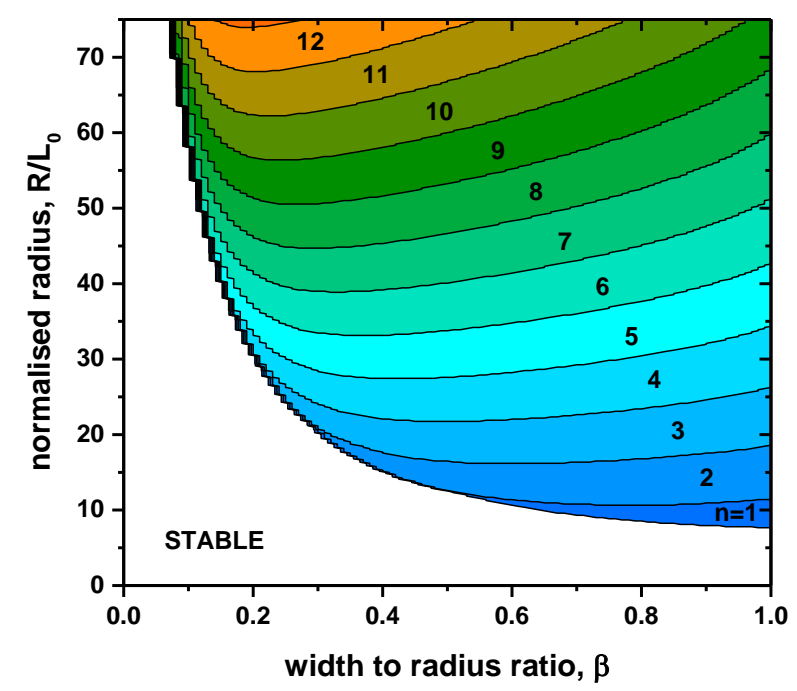

(b)

Figure 4: The optimal mode number $n$, as plotted in Fig. 3a, but for finite slopes with (a) $s_{0}=0.1$, and (b) $s_{0}=0.3$.

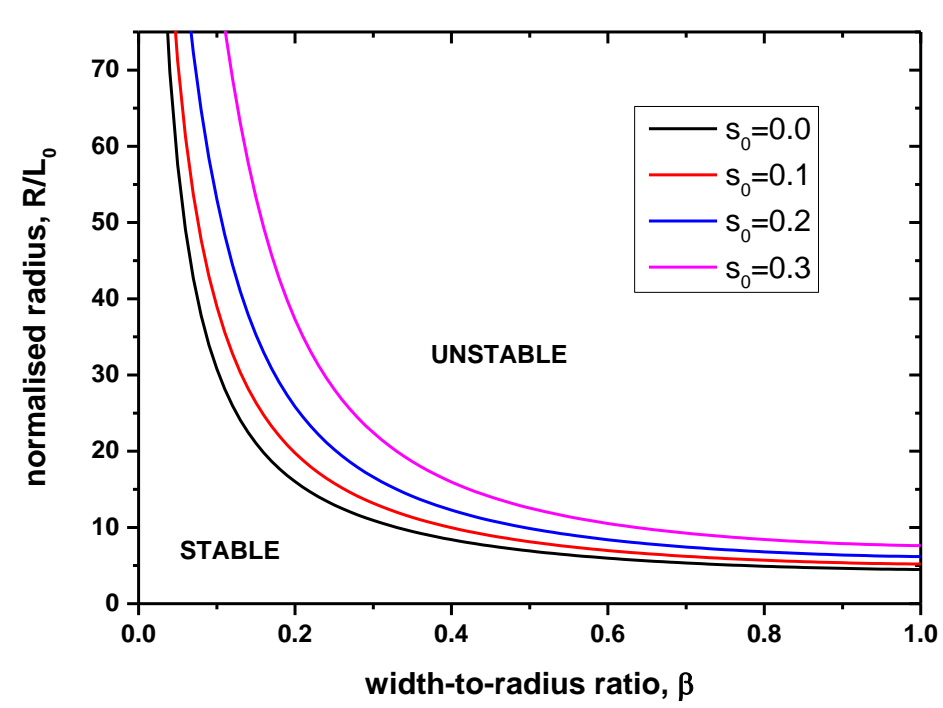

Figure 5: The effect of the nanoring slope $s_{0}$ on the boundary line between the stable and unstable regions

\subsection{Constant volume}

To compare the effect of shape rather than size on the stability of different rings it is useful to investigate their behaviour under the assumption of constant volume. It is assumed that the slope remains constant, with $s_{0}=0.1$, such that a constant reference volume can be defined from (36) as $V_{0}=\frac{8 \pi}{3} s_{0} \beta_{0}^{2} R_{0}^{3}=\frac{8 \pi}{3} s_{0} \beta^{2} R^{3}$, where $R_{0}$ and $\beta_{0}$ prescribe a reference ring structure. To conserve 
volume we now require that $R=\left(\frac{\beta_{0}}{\beta}\right)^{\frac{2}{3}} R_{0}$ such that the radius of the ring expands as the thickness decreases. We take the reference value of $\beta_{0}=0.25$ so the InP rings plotted in Fig. 4 a map onto the same points in constant volume space. We therefore have $\frac{L_{0}}{R}=\frac{L_{0}}{R_{0}}\left(\frac{\beta}{\beta_{0}}\right)^{\frac{2}{3}}$ in equation (49). The relative volume of a nanoring in a particular material system is hence defined by $R_{0} / L_{0}$. The consequent growth rate is plotted in Fig. 6 as a function of $\beta$ and the measure of nanoring volume $R_{0} / L_{0}$. This is essentially a remapping of Fig. 4 such that horizontal lines are now lines of constant volume, under the constant slope assumption. The points for the InP rings at different deposition levels from Fig.4a are shown again in Fig.6a. Travelling horizontally from these points demonstrates how changing the shape of the ring, $\beta$, can affect the resulting QDM without changing the volume of the original ring. Increasing the radius (reducing $\beta$ ) increases the mode number as the larger circumference can accommodate more undulations of the prescribed wavelength. Similarly, reducing the wavelength (increasing $\beta$ ) results in a decrease in the mode number.

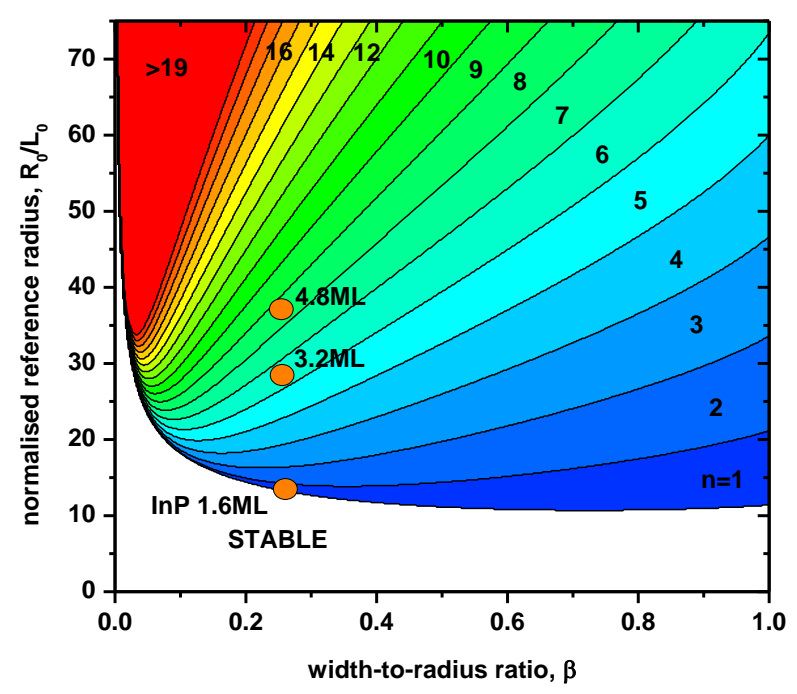

(a) 


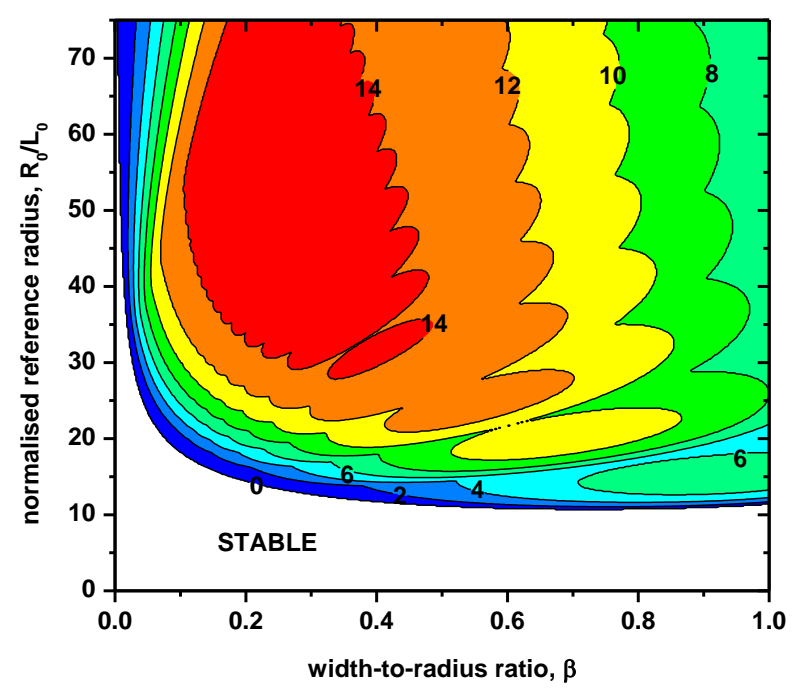

(b)

Fig. 6 : (a) optimal mode number $n$ and (b) optimal growth rate $\bar{\alpha}\left(\times 10^{5}\right)$ for rings with constant slope and variable shape, $\beta$ and $v=0.36$. Fig. $6 a$ is a remapping of Fig. 4 a such that horizontal lines are now contours of constant volume.

Ruffino et al. (2011) investigated the formation of Au nanorings by spontaneous morphological change from pre-exisiting nanoclusters. These rings are different to the model proposed in this paper in the fact that they form with a hole inside the ring which extends below the height of the surrounding substrate. They are generally large rings with radii initially in the range of $100 \mathrm{~nm}$, expanding up to 500nm during annealing. Gill (2012) has shown that this expansion of the rings over time is consistent with the energetics of these structures. They are not observed to break up into QDMs, and it is possible that the internal hole may well be a stabilising influence in this respect. The material properties of the system are approximately $E=79 \mathrm{GPa}, v=0.44$ and $\epsilon_{0}=1.0-1.5 \%$, with $\gamma_{1}=0.8-1.6 \mathrm{~J} / \mathrm{m}^{2}$, so the estimated material length scale is $L_{0}=10-50 \mathrm{~nm}$. During expansion, at the point when $\beta=\beta_{0}$ the radius of the rings is roughly $R_{0}=250 \mathrm{~nm}$. Hence $R_{0} / L_{0}$ is predicted to be between 5 and 25. Unlike the strained droplet epitaxy case, these rings do not grow from a central droplet, and hence there is not a geometrical constraint on the formation of the inner ring radius. Consequently these initial rings can form with very small internal radii, equivalent to $\beta$ in the range 0.7 to 1.0. Fig. 6 a shows that we require $\frac{R_{0}}{L_{0}}<10$ for stability for this range of $\beta$. This is within part of the possible range determined for $R_{0} / L_{0}$, although as stated above, the stability range may be increased by the presence of the central hole in the substrate. Fig $6 a$ also shows that if the initial ring is stable, as it expands ( $\beta$ decreases) it will always stay in the stable zone if its volume remains constant.

\section{Conclusions}

A general non-linear model for the elastic strain energy of non-axisymmetric epitaxially strained nanostructures has been developed. The derivation is beyond the small slope assumption in order to 
properly represent the interactions between the pre-existing nanoring structure and the perturbation. In general the overall energy change can be expanded as

$$
\Delta E=s_{r}^{2}\left[\left(a_{1}+a_{2} s_{r}+a_{3} s_{r}^{2}+\cdots\right)+\left(b_{1}+b_{2} s_{r}+b_{3} s_{r}^{2}+\cdots\right) \eta^{2}+\left(c_{3}+\cdots\right) \eta^{4}\right]
$$

for a ring with pre-existing radial slope $s_{r}$, where the $a_{i}$ etc are shape dependent constants resulting from first order $(i=1)$, second order $(i=2)$ and third order $(i=3)$ terms respectively. This analysis has determined these terms up to second order only, so the $\eta^{4}$ term in (53) is not considered. This is reasonable as it is negligible during the initial stages of the instability and hence is inconsequential in determining the nature of the fastest growing perturbation. The consequences of including such a term are described in detail by Freund and Suresh (2004) for the perturbation of a 2D flat strained film. This shows that the $\eta^{4}$ term can act to halt the growth of the perturbation in the later stages of growth, leading to the creation of a stable, large amplitude undulation. This happens in conjunction with the growth of a second mode. This can be readily introduced in this analysis by extending (1) to the form

$$
\xi(r, t)=h(r)\left[1+\eta_{1}(t) \cos (n \phi)+\eta_{2}(t) \cos (2 n \phi)\right]
$$

where $\eta_{1}$ is the fastest growing mode (simply $\eta$ in this paper) and $\eta_{2}$ is a more slowly growing mode. Subsequent to the initial growth of the perturbation $\eta_{1}$, these two modes can start to interact through the appearance of a second order $\eta_{1}^{2} \eta_{2}$ term in (53). Freund and Suresh (2004) show that in the steady state the second mode is expected to make the troughs of the sinusoidal perturbation slightly sharper and the peaks slightly flatter. These additional terms justify the formation of a final, stable quantum dot molecule, although interactions with the underlying substrate may also be of critical importance in this regard.

The second order model has been used to investigate the stability of strained nanorings subject to perturbations around their circumference evolving via surface diffusion. The fastest growing mode has been related to experimental observations. It is found that the model predicts a region of stability for rings below a critical radius, and also for larger rings which have a proportionally small thickness. The predictions of the model are shown to be consistent with the available results. For the heteroepitaxial InP on $\operatorname{In}_{0.5} G_{0.5} P$ system investigated by Jevasuwan et al. (2013), the nanorings are stable below a certain critical size in good agreement with the model. At larger sizes, the rings are unstable and break up into a number of discrete quantum dots to form a ring-shaped quantum dot molecule. The number of these dots is similar to the mode number for the fastest growing mode. The strained $\mathrm{In}_{0.4} \mathrm{Ga}_{0.6} \mathrm{As}$ on $\mathrm{GaAs}$ nanorings of Hanke et al (2007) are always stable in accordance with the analysis. The Au nanorings of Ruffino et al. (2011) are stable as well, even as they expand during annealing. This observation is also shown to be broadly consistent with the model proposed in this paper. These findings are expected to be useful in the tailoring of heteroepitaxial systems for the self-organisation of quantum dot molecules. 


\section{Appendix A}

Shchukin et al (2004) analysed the elastic relaxation of a conical island on an elastically strained substrate using linear perturbation theory. For a cone of base radius $R_{0}$ and peak height $h_{0}$ they determined the first order relaxation energy to be

$$
\Delta E_{e}^{(1)}=-J(1+v) w_{0} s_{0} V
$$

where the slope is $s_{0}=-h_{0} / R_{0}$, the volume of the cone is $V=\frac{1}{3} \pi s_{0} R_{0}^{3}$ and $J=1.059$. This first order analysis is repeated here using the method outlined in section 2 to check it reproduces this result, and then extended to second order to investigate the effects of large slopes.

The radial and hoop slope components are $s_{r}=s_{0}$ and $s_{\phi}=0$. We have $\beta=1$ and $R=R_{0} / 2$ such that $\bar{s}_{r}=1$. Equations (15) give $P_{r}=\pi s_{0} p_{1}$ and $P_{\phi}=Q_{r}=Q_{\phi}=0$ such that the only non-zero dimensionless term is $\bar{P}_{r}=\pi \bar{p}_{1}$. A non-dimensionalised first order radial displacement is then given by (22) to be

$$
\bar{u}_{r}^{(1)}=-\bar{U}_{r}(\bar{r})=-(1-v) \int_{0}^{2} \bar{p}_{1}\left(\bar{r}, \bar{r}^{\prime}\right) \bar{r}^{\prime} d \bar{r}^{\prime} .
$$

This is plotted in Figure A1 for $v=0.3$. The first and second order changes in elastic strain energy are then given by (33)

$$
\begin{gathered}
\Delta \overline{\mathrm{E}}_{\mathrm{e}}^{(1)}=-\frac{1}{2} \int_{0}^{2} \bar{U}_{r} \bar{r} d \bar{r} \\
\Delta \overline{\mathrm{E}}_{\mathrm{e}}^{(2)}=\frac{1}{(1-v)} \int_{0}^{2} \bar{U}_{r}\left(\bar{r} \frac{d \bar{U}_{r}}{d \bar{r}}+v \bar{U}_{r}\right) d \bar{r}
\end{gathered}
$$

Comparison of (A3a) with (A1) gives

$$
J=\frac{3}{8(1-v)} \int_{0}^{2} \bar{U}_{r} \bar{r} d \bar{r}=1.059
$$

which corresponds exactly with the result of Shchukin et al (2004) as required. Extending this to second order using (A4) we can write $J=1.059+s_{0} \Delta J$ where

$$
\Delta J=\frac{3}{4(1-v)^{2}} \int_{0}^{2} \bar{U}_{r}\left(\bar{r} \frac{d \bar{U}_{r}}{d \bar{r}}+v \bar{U}_{r}\right) d \bar{r}=\left(\frac{2.581 v-0.450}{1-v}\right)
$$

Note that $s_{0}<0$ so the second order correction reduces the expected elastic relaxation for larger slopes. The linear (first order only) and non-linear expressions for $J$ are compared with the results of finite element calculations in Figure A1b. It can be seen that the first order model of Shchukin et al (2004) is valid up to slopes of about 0.15 and that the inclusion of second order terms extends the range of validity up to slopes of about 0.3 .

For completeness, it is of interest to note that for this simple case one can compactly express the second order displacement as

$$
\bar{u}_{r}^{(2)}=-(1-v) \int_{0}^{2} \bar{p}_{1}\left(\bar{r}, \bar{r}^{\prime}\right)\left(\bar{r}^{\prime} \frac{d \bar{U}_{r^{\prime}}}{d \bar{r}^{\prime}}+v \bar{U}_{r^{\prime}}\right) d \bar{r}^{\prime}
$$

This is also shown in Figure A1a. 


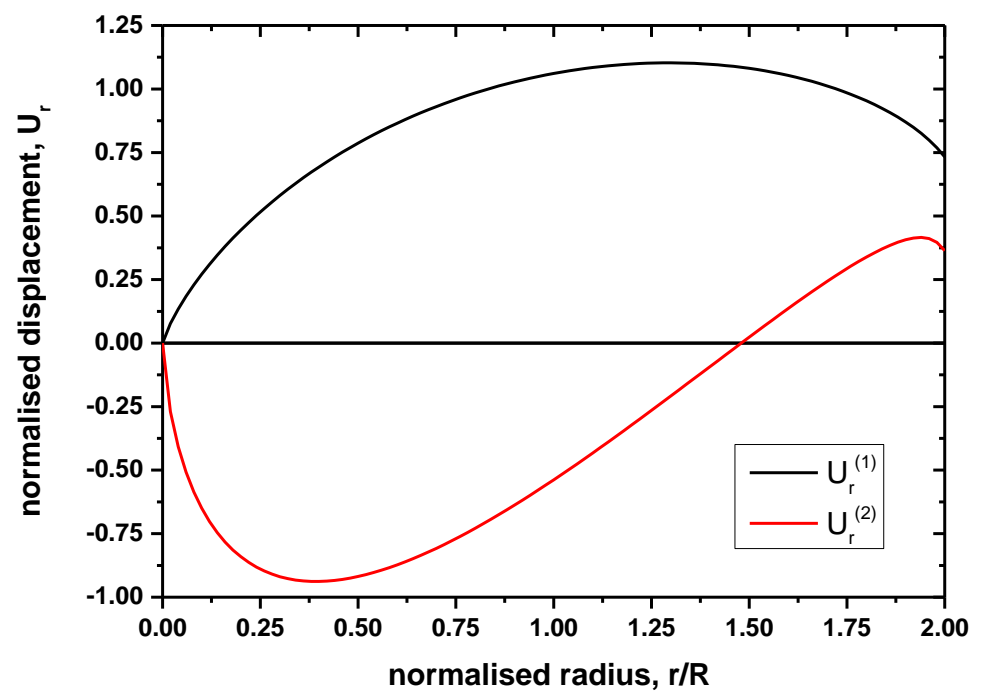

(a)

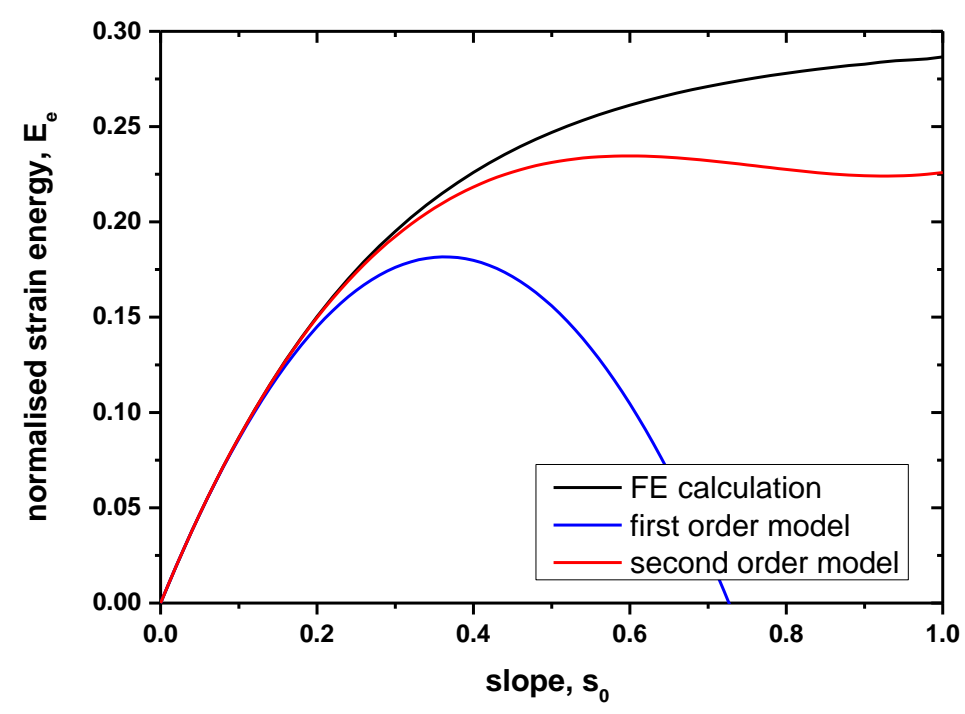

(b)

Figure A1: Elastic relaxation of a conical island. (a) the first and second order components of the radial elastic displacement, and (b) the total elastic relaxation energy calculated using the first and second order models compared with the accurate results of finite element calculations.

\section{References}

Aizpurua, J. , Hanarp, P. , Sutherland, D. S. , Käll, M. , Bryant, G. W. , de Abajo, F. J. G., 2003. Optical Properties of Gold Nanorings. Phys. Rev. Lett. 90, 057401.

Amlani, I., Orlov, A.O., Toth, G., Bernstein, G.H., Lent, C.S., Snider, G.L., 1999. Digital logic gate using quantum-dot cellular automata. Science, 284, 289. 
Cocks, A.C.F. , Gill, S.P.A., Pan, J., 1999. Modelling of microstructural evolution. Adv. Appl. Mech., $36,81$.

Fang, X., Li, Z. , Long, Y. , Wei, H. , Liu, R. , Ma, J. , Kamran, M. , Zhao, H. , Han, X. , Zhao, B., Qiu, X. , 2007. Surface-plasmon-polariton assisted diffraction in periodic sub-wavelength holes of metal films with reduced interplane coupling. Phys. Rev. Lett. 99, 066805.

Freund, L.B, Suresh, S. 2004. Thin film materials : stress, defect formation and surface evolution. (Cambridge University Press).

Gao, H., 1994. Some general properties of stress-driven surface evolution in a heteroepitaxial thin film structure. J. Mech. Phys. Solids 42, 741-772.

Gill, S.P.A., 2003. Self-organised growth on strained substrates: the influence of anisotropic strain, surface energy and surface diffusivity. Thin Solid Films, 423, 136.

Gill, S. P. A., 2009. Enhanced stability of coherently strained conical quantum dot arrays against coarsening: beyond the small slope assumption. Math. Mech. Solids 14, 635.

Gill, S.P.A., 2012. An energetic model for the direct formation and evolution of nanorings. J. Appl. Phys. 113, 154316.

Hanke, M. , Mazur, Yu. I. , Marega Jr., E. , AbuWaar, Z. Y. , Salamo, G. J. , Schäfer, P. , Schmidbauer, M., 2007. Shape transformation during overgrowth of In Ga As Ga As (001) quantum rings. Appl. Phys. Lett. 91, 043103.

He, J. H. , Chen, C. Y. , Ho, C. H. , Wang, C. W. , Chen, M. J. , Chen, L. J., 2010. Growth and structural characterization of SiGe nanorings. J. Phys. Chem. C 114, 5727.

Hobbs, K. L., Larson, P. R. , Lian, G. D. , Keay, J. C. , Johnson, M. B., 2004. Fabrication of nanoring arrays by sputter redeposition using porous alumina templates. Nano Lett. 4, 167.

Hu, H., Gao, H., Liu, F., 2012. Quantitative Model of Heterogeneous Nucleation and Growth of SiGe Quantum Dot Molecules. Phys. Rev. Lett. 109, 106103.

Huang, C. , Ye, J. , Wang, S. , Stakenborg, T. , Lagae, L., 2012. Gold nanoring as a sensitive plasmonic biosensor for on-chip DNA detection. Appl. Phys. Lett.100, 173114.

Jevasuwan, W., Boonpeng, P., Thainoi, S., Panyakeow, S., Ratanathammaphan, S., 2011. InP ringshaped quantum-dot molecules grown by droplet molecular beam epitaxy. J. Crystal Growth 323, 275-278.

Jevasuwan, W. , Ratanathammapan, S. , Panyakeow, S., 2013. InP Ring-Shaped Quantum Dot Molecules by Droplet Epitaxy. Quantum Dot Molecules, Lecture Notes in Nanoscale Science and Technology 14, 29-49 (Springer, J. Wu and Z.M. Wang, eds.).

Kelf, T. A. , Tanaka, Y. , Matsuda, O. , Larsson, E. M. , Sutherland, D. S., Wright, O. B. , 2011. Ultrafast Vibrations of Gold Nanorings. Nano Lett. 11, 3893.

Landau, L. D., Lifshitz, E. M. , 1970. Theory of Elasticity (Pergamon, New York). 
Larsson, E. M. , Alegret, J. , Käll, M. , Sutherland, D. S. 2007. Sensing Characteristics of NIR localized surface plasmon resonances in gold nanorings for application as ultrasensitive biosensors. Nano Lett. $7,1256$.

Lee, J. H. , Sablon, K. , Wang, Zh. M. , Salamo, G. J., 2008. Evolution of InGaAs quantum dot molecules. J. Appl. Phys. 103, 054301.

Li, X.Q., Wu, Y.W., Steel, D., Gammon, D., Stievater, T.H., Katzer, D.S., Park, D., Piermarocchi, C., Sham, L.J., 2003. An all-optical quantum gate in a semiconductor quantum dot. Science 301, 809.

Li, X. L., Yang, G. W. , 2008. Growth mechanisms of quantum ring self-assembly upon droplet epitaxy. J. Phys. Chem. C 112, 7693.

Li, X. L., Yang, G. W., 2009. On the physical understanding of quantum rings self assembly upon droplet epitaxy, J. Appl. Phys. 105, 103507.

Liu, Q. K. K., Moll, N. , Scheffler, M. , Pehlke, E., 1999. Equilibrium shapes and energies of coherent strained InP islands, Phys. Rev. B 60, 17008.

Mishra, Y. K. , Kabiraj, D., Sulania, I. , Pivin, J. C. , Avasthi, D. K., 2007. Synthesis and characterization of gold nanorings. J. Nanosci. Nanotechnol. 7, 1878.

Pang, Y., Huang, R., 2006. Nonlinear effect of stress and wetting on surface evolution of epitaxial thin films. Phys. Rev. B 74, 075413.

Ruffino, F., Crupi, I., Simone, F. , Grimaldi, M. G., 2011. Formation and evolution of self-organized Au nanorings on indium-tin-oxide surface. Appl. Phys. Lett. 98, 023101.

Shchukin, V. A. , Bimberg, D. , Munt, T. P. , Jesson, D. E., 2004. Elastic interaction and self-relaxation energies of coherently strained conical islands. Phys. Rev. B 70, 085416.

Somaschini, C., Bietti, S., Koguchi, N., Sanguinetti, S.,2009. Fabrication of Multiple Concentric Nanoring Structures. Nano Lett. 9, 3419.

Stoffel, M. , Malachias, A. , Rastelli, A. , Metzger, T. H. , Schmidt, O. G. , 2009. Composition and strain in $\mathrm{SiGe} / \mathrm{Si}(001)$ "nanorings" revealed by combined $\mathrm{x}$-ray and selective wet chemical etching methods. Appl. Phys. Lett. 94, 253114.

Sun, Z.-H. , Wang, H. Y. , Zhang, Z.-D. , Zhang, Z.-Y. , 2011. Optical properties of gold nanoring structures. Acta Phys. Sin. 60, 047808.

Suo, Z., 1995. Wrinkling of the oxide scale on an aluminium containing alloy at high temperatures. J. Mech. Phys. Solids. 43, 829.

Wang, Z.M. , Lee, J.H. , Liang, B.L. , Black, W.T. , Kunets, V.P. , Mazur, Y.I., Salamo, G.J. , 2006. Localized formation of InAs quantum dots on shallow-patterned GaAs(100). Appl. Phys. Lett. 88, 233102.

Wang, L. , Montagne, F. , Hoffmann, P. , Heinzelmann, H. , Pugin, R., 2011. Hierarchical positioning of gold nanoparticles into periodic arrays using block copolymer nanoring templates. J. Colloid Int. Sci. $356,496$. 
Yu, L. W. , Chen, K. J. , Song, J. , Xu, J. , Li, W. , Li, X. F. , Wang, J. M. , Huang, X. F. , 2007. New selflimiting assembly model for Si quantum rings on Si(100). Phys. Rev. Lett. 98, 166102.

Zahr, O. K., Blum, A. S. , 2012. Solution phase gold nanorings on a viral protein template. Nano Lett. $12,629$.

Zhou, Z. Y. , Zheng, C. X. , Tang, W. X. , Tersoff, J. , Jesson, D. E., 2013. Origin of quantum ring formation during droplet epitaxy, Phys. Rev. Lett. 111, 036102.

\section{Supplementary material}

The first order elastic relaxation energy of (33a) can also be calculated using the Fourier transform method also adopted by Shchukin et al (2004). This second method is a useful check that the results of (33a) are correct, and was found to be numerically slightly more efficient. Assuming a height profile of the form (1), let the slope in the $x$ and $y$-directions be given by the vector

$$
\boldsymbol{s}(\boldsymbol{r})=\left[\frac{\partial \xi}{\partial r} \cos \phi-\frac{1}{r} \frac{\partial \xi}{\partial \phi} \sin \phi, \frac{\partial \xi}{\partial r} \sin \phi+\frac{1}{r} \frac{\partial \xi}{\partial \phi} \cos \phi\right]
$$

Noting the trigonometric relationships

$$
\begin{aligned}
& 2 \cos n \phi \cos \phi=\cos (n+1) \phi+\cos (n-1) \phi \\
& 2 \cos n \phi \sin \phi=\sin (n+1) \phi-\sin (n-1) \phi \\
& 2 \sin n \phi \cos \phi=\sin (n+1) \phi+\sin (n-1) \phi \\
& 2 \sin n \phi \sin \phi=\cos (n-1) \phi-\cos (n+1) \phi
\end{aligned}
$$

the slope can be expressed in the form

$$
\boldsymbol{s}(\boldsymbol{r})=\left[\begin{array}{c}
A(r) \cos (n+1) \phi+B(r) \cos (n-1) \phi+C(r) \cos \phi \\
A(r) \sin (n+1) \phi-B(r) \sin (n-1) \phi+C(r) \sin \phi
\end{array}\right]
$$

where $A(r)=\frac{\eta}{2}\left(\frac{d h}{d r}-\frac{n h(r)}{r}\right), B(r)=\frac{\eta}{2}\left(\frac{d h}{d r}+\frac{n h(r)}{r}\right)$ and $C(r)=\frac{d h}{d r}$.

The change in the elastic strain energy from the initially flat configuration due to a general surface traction distribution is given by (23)

$$
\Delta E_{e}=-\frac{1}{2} \int f_{i}\left(\boldsymbol{r}^{\prime}\right) u_{i}\left(\boldsymbol{r}^{\prime}\right) d^{2} \boldsymbol{r}^{\prime}=-\frac{1}{2} \iint f_{i}\left(\boldsymbol{r}^{\prime}\right) G_{i j}\left(\boldsymbol{r}-\boldsymbol{r}^{\prime}\right) f_{j}(\boldsymbol{r}) d^{2} \boldsymbol{r} d^{2} \boldsymbol{r}^{\prime}
$$

or

$$
\Delta E_{e}=-\frac{(1+v) w_{0}}{2 \pi(1-v)} \int_{A} d^{2} \boldsymbol{r} \int_{A} d^{2} \boldsymbol{r}^{\prime} s_{i}(\boldsymbol{r})\left[\frac{(1-v) \delta_{i j}}{\left|\boldsymbol{r}-\boldsymbol{r}^{\prime}\right|}-\frac{v\left(\boldsymbol{r}-\boldsymbol{r}^{\prime}\right)_{i}\left(\boldsymbol{r}-\boldsymbol{r}^{\prime}\right)_{j}}{\left|\boldsymbol{r}-\boldsymbol{r}^{\prime}\right|^{3}}\right] s_{j}\left(\boldsymbol{r}^{\prime}\right)
$$

Following Shchukin et al (2004) and Gill (2009), the form of the expression (1) allows separation of the radial and angular components of (S5) such that

$$
\Delta E_{e}=-\frac{(1+v) w_{0}}{2 \pi(1-v)} \int_{0}^{\infty} \int_{0}^{\infty} r r^{\prime} J\left(r, r^{\prime}\right) d r d r^{\prime}
$$


where

$$
J\left(r, r^{\prime}\right)=\int_{0}^{2 \pi} \int_{0}^{2 \pi} s_{i}(\boldsymbol{r})\left[\frac{(1-v) \delta_{i j}}{\left|\boldsymbol{r}-\boldsymbol{r}^{\prime}\right|}+\frac{v\left(\boldsymbol{r}-\boldsymbol{r}^{\prime}\right)_{i}\left(\boldsymbol{r}-\boldsymbol{r}^{\prime}\right)_{j}}{\left|\boldsymbol{r}-\boldsymbol{r}^{\prime}\right|^{3}}\right] s_{j}\left(\boldsymbol{r}^{\prime}\right) d \phi d \phi^{\prime}
$$

We use the identity

$$
\frac{\left(\boldsymbol{r}-\boldsymbol{r}^{\prime}\right)_{i}\left(\boldsymbol{r}-\boldsymbol{r}^{\prime}\right)_{j}}{\left|\boldsymbol{r}-\boldsymbol{r}^{\prime}\right|^{3}}=\frac{\delta_{i j}}{\left|\boldsymbol{r}-\boldsymbol{r}^{\prime}\right|}-\nabla_{i} \nabla_{j}\left|\boldsymbol{r}-\boldsymbol{r}^{\prime}\right|
$$

such that (S7) becomes

$$
J\left(r, r^{\prime}\right)=\int_{0}^{2 \pi} \int_{0}^{2 \pi} s_{i}(\boldsymbol{r})\left[\frac{\delta_{i j}}{\left|\boldsymbol{r}-\boldsymbol{r}^{\prime}\right|}-v \nabla_{i} \nabla_{j}\left|\boldsymbol{r}-\boldsymbol{r}^{\prime}\right|\right] s_{j}\left(\boldsymbol{r}^{\prime}\right) d \phi d \phi^{\prime} .
$$

Expressed in Fourier space this is

$$
J\left(r, r^{\prime}\right)=\frac{1}{(2 \pi)^{2}} \int d^{2} \boldsymbol{k}\left[\tilde{S}_{i}(\boldsymbol{k} ; r)\right]^{*} \tilde{G}_{i j}(\boldsymbol{k}) \tilde{S}_{j}\left(\boldsymbol{k} ; \boldsymbol{r}^{\prime}\right)
$$

where Shchukin et al (2004) show that the Fourier transform of the Green's tensor is

$$
\tilde{G}_{i j}(\boldsymbol{k})=\frac{2 \pi}{k}\left(\delta_{i j}-v \frac{k_{i} k_{j}}{k^{2}}\right)
$$

and the Fourier transform of the slope has the normal definition

$$
\tilde{S}_{i}(\boldsymbol{k} ; r)=\int_{0}^{2 \pi} s_{i}(r, \phi) e^{-i k r \cos (\phi-\psi)} d \phi
$$

where $\psi$ is the polar angle in $\boldsymbol{k}$-space, such that $\boldsymbol{k}=k[\cos \psi, \sin \psi]$. From (S3), the transform (S12) consists of terms of the type

$$
\begin{aligned}
& \int_{0}^{2 \pi} \cos m \phi e^{-i k r \cos (\phi-\psi)} d \phi=\int_{0}^{2 \pi} \cos m(\chi+\psi) e^{-i k r \cos \chi} d \chi \\
& =\cos (m \psi) \int_{0}^{2 \pi} \cos (m \chi) e^{-i k r \cos \chi} d \chi-\sin (m \psi) \int_{0}^{2 \pi} \sin (m \chi) e^{-i k r \cos \chi} d \chi
\end{aligned}
$$

where $\chi=\phi-\psi$. The second integral in (S13b) is zero due to the odd terms, and the first integral defines a Bessel function of the first kind, such that

$$
\int_{0}^{2 \pi} \cos m \phi e^{-i k r \cos (\phi-\psi)} d \phi=2 \pi(-i)^{m} \cos (m \psi) J_{m}(k r) .
$$

Similarly

$$
\int_{0}^{2 \pi} \sin m \phi e^{-i k r \cos (\phi-\psi)} d \phi=2 \pi(-i)^{m} \sin (m \psi) J_{m}(k r)
$$

Using (S3), one can therefore express (S12) as

$$
\tilde{\boldsymbol{S}}(\boldsymbol{k} ; r)=\left[\begin{array}{c}
\tilde{A} \cos (n+1) \psi+\tilde{B} \cos (n-1) \psi+\tilde{C} \cos \psi, \\
\tilde{A} \sin (n+1) \psi-\tilde{B} \sin (n-1) \psi+\tilde{C} \sin \psi
\end{array}\right]
$$

where $\tilde{A}(k, r)=2 \pi(-i)^{n+1} A(r) J_{n+1}(k r), \tilde{B}(k, r)=2 \pi(-i)^{n-1} B(r) J_{n-1}(k r)$ and $\tilde{C}(k, r)=$ $-2 \pi i C(r) J_{1}(k r)$ are functions of $k$ and $r$. 
We can now express (S10) as

$$
J\left(r, r^{\prime}\right)=\frac{1}{(2 \pi)^{2}} \int_{0}^{\infty} \int_{0}^{2 \pi}\left[\tilde{S}_{i}(\boldsymbol{k} ; r)\right]^{*} \frac{2 \pi}{k}\left(\delta_{i j}-v \frac{k_{i} k_{j}}{k^{2}}\right) \tilde{S}_{j}\left(\boldsymbol{k} ; r^{\prime}\right) d \psi k d k
$$

The integrals over $\psi$ are trivial, such that, for the $\delta_{i j}$ term, we have for $n>0$

$$
\int_{0}^{2 \pi}\left[\tilde{S}_{i}(\boldsymbol{k} ; r)\right]^{*} \delta_{i j} \tilde{S}_{j}\left(\boldsymbol{k} ; r^{\prime}\right) d \psi=2 \pi\left[\tilde{A}^{*}(k, r) \tilde{A}\left(k, r^{\prime}\right)+\tilde{B}^{*}(k, r) \tilde{B}\left(k, r^{\prime}\right)+\tilde{C}^{*}(k, r) \tilde{C}\left(k, r^{\prime}\right)\right]
$$

and the second term in the integrand of (S17) gives

$$
\begin{gathered}
\int_{0}^{2 \pi}\left[\tilde{S}_{i}(\boldsymbol{k} ; r)\right]^{*} \frac{k_{i} k_{j}}{k^{2}} \tilde{S}_{j}\left(\boldsymbol{k} ; r^{\prime}\right) d \psi=\pi\left[\tilde{A}^{*}(k, r) \tilde{A}\left(k, r^{\prime}\right)+\tilde{B}^{*}(k, r) \tilde{B}\left(k, r^{\prime}\right)+\tilde{A}^{*}(k, r) \tilde{B}\left(k, r^{\prime}\right)\right. \\
\left.+\tilde{B}^{*}(k, r) \tilde{A}\left(k, r^{\prime}\right)+2 \tilde{C}^{*}(k, r) \tilde{C}\left(k, r^{\prime}\right)\right]
\end{gathered}
$$

Note that the expressions (S18) and (S19) contain no interactions between the mode 0 term $(\tilde{C})$ and the mode $n$ terms $(\tilde{A}$ and $\tilde{B})$. Careful examination shows that there are no interactions between any of the different modes to first order, and hence the above analysis can be readily used to derive the elastic strain energy for a general Fourier series expression for $g(\phi)$.

Hence (S17) can be written as

$$
\begin{gathered}
J\left(r, r^{\prime}\right)=\frac{2 \pi}{(2 \pi)^{2}} \int_{0}^{\infty} \frac{2 \pi}{k}\left[(1-v) \tilde{C}^{*}(k, r) \tilde{C}\left(k, r^{\prime}\right)+\left(1-\frac{1}{2} v\right)\left(\tilde{A}^{*}(k, r) \tilde{A}\left(k, r^{\prime}\right)+\tilde{B}^{*}(k, r) \tilde{B}\left(k, r^{\prime}\right)\right)\right. \\
\left.-\frac{1}{2} v\left(\tilde{A}^{*}(k, r) \tilde{B}\left(k, r^{\prime}\right)+\tilde{B}^{*}(k, r) \tilde{A}\left(k, r^{\prime}\right)\right)\right] k d k
\end{gathered}
$$

or

$$
\begin{gathered}
J\left(r, r^{\prime}\right)=(1-v)(2 \pi)^{2} C(r) C\left(r^{\prime}\right) \int_{0}^{\infty} J_{1}(k r) J_{1}\left(k r^{\prime}\right) d k \\
+\left(1-\frac{1}{2} v\right)(2 \pi)^{2}\left[A(r) A\left(r^{\prime}\right) \int_{0}^{\infty} J_{n+1}(k r) J_{n+1}\left(k r^{\prime}\right) d k+B(r) B\left(r^{\prime}\right) \int_{0}^{\infty} J_{n-1}(k r) J_{n-1}\left(k r^{\prime}\right) d k\right] \\
+\frac{1}{2} v(2 \pi)^{2}\left[A(r) B\left(r^{\prime}\right) \int_{0}^{\infty} J_{n+1}(k r) J_{n-1}\left(k r^{\prime}\right) d k+B(r) A\left(r^{\prime}\right) \int_{0}^{\infty} J_{n-1}(k r) J_{n+1}\left(k r^{\prime}\right) d k\right]
\end{gathered}
$$

Gradshteyn and Ryzhik (1980) give the following expressions for the Bessel function integrals

$$
\int_{0}^{\infty} J_{m}(k r) J_{m}\left(k r^{\prime}\right) d k=\frac{\Gamma\left(m+\frac{1}{2}\right)}{\Gamma(m+1) \Gamma\left(\frac{1}{2}\right)}\left(\frac{r_{<}^{m}}{r_{>}^{m+1}}\right){ }_{2} F_{1}\left(m+\frac{1}{2}, \frac{1}{2} ; m+1 ;\left(\frac{r_{<}}{r_{>}}\right)^{2}\right)
$$

where $r_{<}=\min \left(r, r^{\prime}\right)$ and $r_{>}=\max \left(r, r^{\prime}\right),{ }_{2} F_{1}(a, b ; c ; x)$ is the hypergeometric function, and

$$
\begin{aligned}
& \int_{0}^{\infty} J_{m+1}\left(k r_{>}\right) J_{m-1}\left(k r_{<}\right) d k=\frac{\Gamma\left(m+\frac{1}{2}\right)}{\Gamma(m) \Gamma\left(\frac{3}{2}\right)}\left(\frac{r_{<}^{m-1}}{r_{>}^{m}}\right){ }_{2} F_{1}\left(m+\frac{1}{2},-\frac{1}{2} ; m ;\left(\frac{r_{\leq}}{r_{>}}\right)^{2}\right) \\
& \int_{0}^{\infty} J_{m-1}\left(k r_{>}\right) J_{m+1}\left(k r_{<}\right) d k=\frac{\Gamma\left(m+\frac{1}{2}\right)}{\Gamma(m+2) \Gamma\left(-\frac{1}{2}\right)}\left(\frac{r_{<}^{m+1}}{r_{>}^{m+2}}\right){ }_{2} F_{1}\left(m+\frac{1}{2}, \frac{3}{2} ; m+2 ;\left(\frac{r_{\leq}}{r_{>}}\right)^{2}\right)
\end{aligned}
$$

The strain energy (S6) can be written in terms of $r_{<}$and $r_{>}$as 


$$
\Delta E_{e}=-\frac{(1+v) w_{0}}{2 \pi(1-v)} 2 \int_{0}^{\infty} r_{>} \int_{0}^{r_{>}} r_{<} J\left(r_{<}, r_{>}\right) d r_{<} d r_{>}
$$

Inserting the expression (S21), with the definitions (S23), this can finally be written as

$$
\Delta E_{e}=-\frac{2 \pi(1+v) w_{0}}{(1-v)}\left[(1-v) I_{c c}+\left(1-\frac{1}{2} v\right)\left(I_{A A}+I_{B B}\right)+\frac{1}{2} v\left(I_{A B}+I_{B A}\right)\right]
$$

where

$$
\begin{aligned}
& I_{A A}=2 \frac{\Gamma\left(n+\frac{3}{2}\right)}{\Gamma(n+2) \Gamma\left(\frac{1}{2}\right)} \int_{0}^{\infty} \int_{0}^{r_{>}} A\left(r_{<}\right) A\left(r_{>}\right)\left(\frac{r_{<}^{n+1}}{r_{>}^{n+2}}\right){ }_{2} F_{1}\left(n+\frac{3}{2}, \frac{1}{2} ; n+2 ;\left(\frac{r_{<}}{r_{>}}\right)^{2}\right) r_{<} r_{>} d r_{<} d r_{>} \\
& I_{B B}=2 \frac{\Gamma\left(n-\frac{1}{2}\right)}{\Gamma(n) \Gamma\left(\frac{1}{2}\right)} \int_{0}^{\infty} \int_{0}^{r_{>}} B\left(r_{<}\right) B\left(r_{>}\right)\left(\frac{r_{<}^{n-1}}{r_{>}^{n}}\right){ }_{2} F_{1}\left(n-\frac{1}{2}, \frac{1}{2} ; n ;\left(\frac{r_{<}}{r_{>}}\right)^{2}\right) r_{<} r_{>} d r_{<} d r_{>} \\
& I_{A B}=2 \frac{\Gamma\left(n+\frac{1}{2}\right)}{\Gamma(n+2) \Gamma\left(-\frac{1}{2}\right)} \int_{0}^{\infty} \int_{0}^{r_{>}} A\left(r_{<}\right) B\left(r_{>}\right)\left(\frac{r_{<}^{n+1}}{r_{>}^{n+2}}\right){ }_{2} F_{1}\left(n+\frac{1}{2}, \frac{3}{2} ; n+2 ;\left(\frac{r_{<}}{r_{>}}\right)^{2}\right) r_{<} r_{>} d r_{<} d r_{>} \\
& I_{B A}=2 \frac{\Gamma\left(n+\frac{1}{2}\right)}{\Gamma(n) \Gamma\left(\frac{3}{2}\right)} \int_{0}^{\infty} \int_{0}^{r_{>}} B\left(r_{<}\right) A\left(r_{>}\right)\left(\frac{r_{<}^{n-1}}{r_{>}^{n}}\right){ }_{2} F_{1}\left(n+\frac{1}{2},-\frac{1}{2} ; n ;\left(\frac{r_{<}}{r_{>}}\right)^{2}\right) r_{<} r_{>} d r_{<} d r_{>} \\
& I_{C C}=2 \frac{\Gamma\left(\frac{3}{2}\right)}{\Gamma(2) \Gamma\left(\frac{1}{2}\right)} \int_{0}^{\infty} \int_{0}^{r_{>}} C\left(r_{<}\right) C\left(r_{>}\right)\left(\frac{r_{<}}{r_{>}^{2}}\right){ }_{2} F_{1}\left(\frac{3}{2}, \frac{1}{2} ; 2 ;\left(\frac{r_{<}}{r_{>}}\right)^{2}\right) r_{<} r_{>} d r_{<} d r_{>}
\end{aligned}
$$

Hence it is possible to express the strain energy (S25) as

$$
\Delta E_{e}=-\left(E_{e 0}+\eta^{2} E_{e n}\right)
$$

where $E_{e 0}$ is the mode 0 term (strain relaxation energy due to the formation of the initial nanoring) to which only the $I_{C C}$ integral contributes, and $E_{e n}$ is the mode $n$ term (change in strain energy due to the undulation) which arises from the remainder. These contributions are readily determined from the integrals in (S26) given the initial geometry of the nanoring. The dimensionless variable, $\eta(t)$, represents the amplitude of the undulation and is shown explicitly as it is the only timedependent quantity. The two energy terms can be calculated using the series expansion definition of the hypergeometric functions

$$
{ }_{2} F_{1}(a, b ; c ; x)=\sum_{p=0}^{\infty} \frac{\Gamma(p+a) \Gamma(p+b) \Gamma(c)}{\Gamma(a) \Gamma(b) \Gamma(p+c) \Gamma(p+1)} x^{p}
$$

where $\Gamma(p)$ is the Gamma function. If $h(r)$ is a polynomial then the integrations are trivial. However, the expressions are messy so this has been done using the mathematical package Maple. The first 250 terms in the series are considered to achieve good convergence. Importantly, the numerical accuracy of the calculation must be increased (to 20 digits) to ensure proper convergence. From (S27) one can write the important mode $n$ terms in non-dimensional form as

$$
\bar{E}_{e n}(n, \beta, v)=\left(1-\frac{1}{2} v\right)\left(\bar{I}_{A A}+\bar{I}_{B B}\right)+\frac{1}{2} v\left(\bar{I}_{A B}+\bar{I}_{B A}\right)
$$

and the dimensionless $\bar{I}$ functions have been derived from (S26) such that, for instance 


$$
\bar{I}_{A A}(n, \beta)=2 \frac{\Gamma\left(n+\frac{3}{2}\right)}{\Gamma(n+2) \Gamma\left(\frac{1}{2}\right)} \int_{1-\beta}^{1+\beta} \int_{1-\beta}^{\rho_{>}} \bar{A}\left(\rho_{<}\right) \bar{A}\left(\rho_{>}\right)\left(\frac{\rho_{<}^{n+1}}{\rho_{>}^{n+2}}\right){ }_{2} F_{1}\left(n+\frac{3}{2} ; \frac{1}{2} ; n+2 ;\left(\frac{\rho_{<}}{\rho_{>}}\right)^{2}\right) \rho_{<} \rho_{>} d \rho_{<} d \rho_{>}
$$

with $\rho=r / R$, and $\bar{A}(\rho)=\frac{1}{2}\left(\frac{d \bar{h}}{d \rho}-\frac{n \bar{h}(\rho)}{\rho}\right)$ etc. The dimensionless height profile is taken to be $\bar{h}(\rho)=1-\frac{(1-\rho)^{2}}{\beta^{2}}$.

\section{Reference}

Gradshteyn, I. S., Ryzhik, I. M., 1980. Tables of Integrals, Series, and Products (Academic Press, New York). 